\title{
ILFCS: an intelligent learning fuzzy-based channel selection framework for cognitive radio networks
}

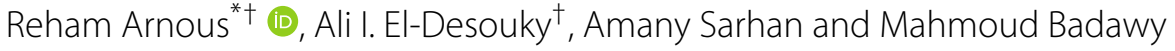

\begin{abstract}
Cognitive radio networks (CRNs) have been introduced as a promising solution to optimize the use of available radio-frequency spectrum. The key idea in CRNs is the proper selection of available sensed channels. In this paper, an intelligent distributed channel selection strategy is proposed for cognitive radio ad-hoc networks aiming to assist them in selecting the best channel for transmission. The proposed strategy classifies the available channels based on the primary users'(PUs) utilization, the number of cognitive radio neighbors using the channels, and the capacity of available channels. The Fuzzy Logic technique is used to determine a channel's weight value by combining these parameters. The channels with the highest weight value are selected for transmission. The proposed strategy takes into account false alarm (FA) and miss detection (MD) metrics to classify the sensed channels into four categories (FA, MD, ON and OFF) based on $K$-means learner. This classification helps the strategy to avoid accessing occupied channels. Simulation results based on NS2 simulation approved that the proposed strategy is effective compared to other strategies concerning selecting the best channel and achieving higher channel utilization.
\end{abstract}

Keywords: Channel selection, Cognitive radio networks, Fuzzy logic, Spectrum characterization, Spectrum Decision

\section{Introduction}

Conventionally, the radio frequency (RF) spectrum is regulated by assigning fixed portions of spectrum to individual users in the form of renewable licenses. However, the fixed spectrum assignment policy ensures interferencefree communications between radio terminals that cause poor spectrum utilization. Recent studies of the Federal Communications Commission (FCC) showed that spectrum utilization of licensed RF bands varies from 15 to $85 \%$ based on the location and the time. The rapid growth of wireless device applications increases the demand of the electromagnetic radio spectrum. Consequently, waste of spectrum resources has become a serious problem. To address this problem, cognitive radio networks (CRNs) have appeared as a promising solution [1]. In these networks, the unlicensed cognitive radio users (CRs) are allowed to opportunistically access the licensed spectrum without interfering with licensed primary users (PUs).

\footnotetext{
*Correspondence: rehamarnous@gmail.com

${ }^{\dagger}$ Reham Arnous and Ali I. El-Desouky contributed equally to this work. ${ }^{1}$ Department of Computer Eng. and Control Sys., Faculty of Engineering Mansoura University, Masoura, Egypt
}

CRs are characterized by cognitive radio (CR) capabilities, which enable them to sense the spectrum environment, to detect available channels in wireless spectrum, and to change their transmission and reception parameters accordingly. CRs can use the licensed spectrum as long as the PU is absent while vacate it if primary user comes back into operation. This optimizes the use of available RF spectrum while minimizing interference with the primary users [2].

Achieving efficient spectrum utilization in CRNs requires four successive management procedures: spectrum sensing, spectrum decision, spectrum sharing, and spectrum mobility $[3,4]$. First, CRs have to continually monitor the radio spectrum in order to find the spectrum holes that are unemployed by the primary users through spectrum sensing operation. Reliable spectrum sensing is perceived as a vital part of the CR networks [5].The accuracy of spectrum sensing is evaluated by means of the two probabilities, the miss detection probability and the false alarm probability. A false alarm incident occurs when CR user claims that the channel is busy whereas its actual state 
is idle. However, a miss detection incident happens when CR user fails to detect the presence of PUs $[6,7]$.

Different sensing techniques like matched filter, energy, and feature detection were used [8]. However, many factors such as channel fading, shadowing effects, and receiver uncertainty cause the inaccurate detection of PUs' activities leading to miss detection problems [9]. Cooperative spectrum sensing approach improves PUs' detection by utilizing spectrum sensing information from other CRs. CR users share their sensing information to make more precise decision. The channel is identified as idle if all CRs decide this. Though miss detection probability is decreased, the false alarm probability is increased, which in turn drops the spectrum utilization $[10,11]$.

The spectrum bands that are detected through sensing have to be investigated to define their characteristics. Each CR user should select the most appropriate band that satisfies its transmission requirements and simultaneously avoid interference with primary users. Then, the transmission parameters are reconfigured in order to support communication within the selected band. Spectrum characterization, spectrum selection, and CR reconfiguration functions are the three main functions of spectrum decision process [12]. In CRNs, spectrum decision is a key function that ensures the maximum spectrum utilization and the best transmission performance [13, 14].

This paper proposes a new spectrum selection framework for the cognitive radio wireless ad hoc network (CRAHN) called an intelligent learning fuzzy-based channel selection (ILFCS) framework. The proposed ILFCS aims at overcoming the problem of sensing errors that reduces the performance of CRNs and introducing a new channel selection scheme to help CR users select the best channel in an intelligent way. Actually, the ILFCS framework enhances the network performance by decreasing false alarm and miss detection effects and enabling CRs to select the best channel, which improves their transmission performance and maximizes the throughput and the spectrum utilization. This enhancement is based on two contributions, namely, the use of $K$-means unsupervised learning technique to minimize the sensing errors, and the application of the fuzzy inference system (FIS) as a channel selection strategy to select the best channel in an intelligent process. ILFCS is evaluated by the NS2 simulator. The simulation results show that this approach ensures selecting best channel with less PUs' interference compared to related strategies.

The remainder of this paper is organized as follows: Section 2 surveys related work. The motivations and contributions are listed in Section 3. Section 4 describes the system model. Section 5 explains the proposed ILFCS framework and illustrates the $K$-means learner and fuzzybased channel selector modules. Section 6 introduces the simulation results and the performance comparison of the proposed framework to other strategies, and finally, this work is concluded in Section 7.

\section{Related work}

Convenient selection of channel is the most vital task in cognitive radio. Recently, a lot of work is done on channel selection in cognitive radio ad hoc network (CRAHN). Various channel selector strategies have been used in the current research works. Q-Learning has been applied to channel selection in order to enhance CRs' performance and at the same time to minimize interference to PUs.

Tang et al. [15] select the channel with low PUs' activities aiming to minimize the channel switching delay of each CR node. In another paper by Li [16], each CR user learns how to select channel according to its experience by applying multi-agent reinforcement learning (MARL). He et al. [17] proposed a joint channel selection and power control spectrum decision algorithm based on distributed Q learning where CRs select optimal channel which guarantee maximum energy and spectrum efficiency.

Game theory is also introduced for channel allocation in CRNs where each single agent acts as an independent decision maker. The CRs are the players which select the transmission channel and the related transmission parameters. Gállego et al. presented channel allocation and power control strategies in CRNs using game theory aiming to maximize network utility in which the problem has been analyzed under the physical interference model in which a link can only be established if the received SINR is higher than a predefined threshold [18]. Salim et al. proposed an energy-efficient game-theory-based spectrum decision (EGSD) scheme for CR networks to increase the network lifetime [19]. Graph Coloring based Dynamic Channel Allocation (GC-DCA) algorithm is proposed that minimizes interference with PUs and increases channel utilization [20].

There are other different scenarios introduced for channel selection in CR network. Ding et al. proposed a distributed algorithm for joint opportunistic routing and dynamic spectrum access in multi hop CRNs [21]. Pareek et al. proposed a cluster-based channel assignment scheme for CRNs [22]. Weiwei and Xiaohui suggested a robust resource allocation (RA) algorithm for cognitive relay networks with multiple PUs. The proposed algorithm solved the resource allocation through robust relay selection and power allocation distinctly bearing in mind both channel and interference uncertainty. Results verified the robustness of the suggested robust relay selection scheme [23].

Veeramakali et al. recommended an intelligent fuzzybased dynamic spectrum allocation (IDSA) with bandwidth flexibility for $\mathrm{CR}$. The suggested strategy achieved an appreciable performance enhancement taking into 
consideration three measures: service rate, average packet loss, and average delay [24]. López et al. solve the problem of spectrum allocation regarding the criteria of equity by enabling all the cognitive radio nodes to access the available network resources in a reasonable way without monopolizing the current capacity. The CR nodes are classified based on the network usage historical metrics. Nodes with better ranking have higher priority in the spectrum allocation. Two techniques (ANFIS and FAHP) are used for categorizing. Results of each technique are compared to each other and to a channel assignment algorithm that does not perform any classification regarding a new measure of quantifiable fairness to measure system performance [25]. EElhachmi et al. applied a dynamic genetic algorithm (GA) for channel allocation in cognitive radio. Their suggested algorithm, which is based on the new sophisticated crossover and mutation operators, provides an efficient way for the available spectrum allocation for both primary and secondary users [26]. Suzan et al. introduced the best fit channel selection (BFC) for distributed channel selection. BFC strategy takes into account both the primary channel traffic activity and the CR traffic activity in channel selection. CR nodes can estimate PUs' traffic activities, can determine the channel state and can predict the time the channel stays in the idle state. Consequently, it selects the channels with longer idle time than the time required for completing its transmission [27]. In the longest idle time channel selection (LITC), a CR user simply selects the channel that has the longest expected idle time ignoring its transmission needs. It is a selfish approach that can passively affect the entire network $[28,29]$.

Tarek et al. proposed an intelligent channel selection scheme for cognitive radio ad hoc networks (ICSSSS) using a self-organized map followed by a simple segregation. The channels are classified into four clusters by using SOM learner that helps CR to select the available channels by minimizing the sensing error. Thus, the best one is selected for secondary users out of these channels through using a simple segregated channel selector in a reasonable time [30].

For the best of our knowledge, all the mentioned methods ignored the impact of sensing errors (e.g., false alarm and miss detection) in the channel selection process. As a result, the hopping rate of a CR to move from a channel to another increased. In addition, CRs frequently disturb PUs. Finally, this ends up with poor throughput and channel utilization. To solve this problem, the new efficient channel decision framework (ILFCS) that takes into account the sensing error probabilities is proposed for CRAHNs. The new ILFCS is based on a wellknown unsupervised learning technique, $K$-means, which enables CR nodes to avoid selecting a wrong channel. In addition, fuzzy inference system procedure permits an improved tuning of the accurate predictions. Also, it allows all CR nodes to utilize all available channels correctly.

\section{Motivation and contributions}

In this paper, we focus on channel selection in CRAHNs, where CRs have to compete with the PUs for the residual resources on channels in order to use them opportunistically. Due to the nature of the CRAHN, channel selection decision increases basic challenges regarding the implementation of effective distributed schemes. In CRAHN, chosen reliability channel is difficult to be achieved because of many factors as follows:

- In addition to the already known issues of wireless environments, the channel availability, which is determined by the present behavior of PUs, adds a noticeable load on CRAHN.

- The switching to other available channels once the PU appears on the occupied channel makes the CR user loses its connection. Consequently, the mobility of users involved in an on-going communication drives the CR user to sustain the quality of service (QoS) or to minimize the quality degradation during the spectrum switching through interacting with each layering protocol. Thus, the process of switching from a channel to another needs to be minimized by the accurate prediction of the PU traffic.

- The inaccurate detection of PU activities, which is caused by spectrum sensing errors (sum of MD and FA), increases collision possibilities with PUs. So avoiding FA and MD is a major challenge in CRNs.

- Selecting the most proper channel that achieves the least interference between CRs' flow and each other creates another issue in CRNs.

The proposed ILFCS takes into account all abovementioned issues. The general objectives of ILFCS can be summarized as follows:

- Improving the accuracy in selecting the best channel over a CRAHN to help CRs to satisfy its QoS requirements.

- Minimizing sensing errors resulted from the spectrum sensing techniques and average end-to-end delay over the whole CRNs.

- Avoiding channel conflict among CR users.

- Improving spectrum channel utilization and reducing the interference ratio between CRs and Pus.

- Comparing the proposed ILFCS framework and other related strategies. The simulation results demonstrated that the proposed framework outperforms other strategies in selecting best channel with less PUs' interference and achieving high spectrum utilization. 


\section{System model}

In this section, the considered system model and the basic related assumptions to the proposed selection framework are presented.

\subsection{Network model}

The network consists of a group of both primary radio nodes and cognitive radio nodes. Primary radio nodes are the licensed users who have the highest priority to access the channels and who should not be interrupted by the CR node transmissions. This paper will take into account a distributed primary network that uses the time slotted access method. The PUs are supposed to follow the ON/OFF activity model.

We consider a cognitive radio ad hoc network where no centralized network entity is available. Network operations (e.g., spectrum sensing, channel selection decision) are performed by the CR nodes themselves [31]. We further assume that CR nodes have the same capability of PU nodes. The main difference between them is that PU has an owner band which can be used anywhere, while the CR can only access the idle frequency band. If a signal from a PU is detected, the CR user must vacate the allocated spectrum. CRs are supposed to be provided with a single transceiver where the $\mathrm{CR}$ either sense or communicate on one channel at a time. This reduces the computational cost of the CRs and avoids the likely interference when using multi-transceiver [32, 33].

As CRAHNs do not depend on any predefined infrastructure backbone, $\mathrm{CR}$ users communicate with each other in ad hoc manner. One common control channel (CCC) is supposed to be available for secondary cognitive users to exchange all messages required for coordination and sharing of their sensing results. Thus, each CR user has complete knowledge of vacant channels available found in the PU band after synchronization. It is worth saying that CRAHN is considered a more suitable option due to the advantages of having a lower cost of implementation, lower system complexity, and faster align positioning compared to the infrastructure-based CRN $[34,35]$.

CRNs are supposed to work in a standalone fashion and make decisions based on locally inferred information. Therefore, each CR node has to perform spectrum sensing to detect the existence of the PU signal. The spectrum sensing is periodically performed by every CR node. In the proposed ILFCS, the spectrum sensing block is suggested to be responsible for the detection of the PU signal. The proposed ILFCS will work on the list of available channels resulting from the spectrum sensing. The currently used spectrum sensing techniques involves sensing errors that affect the performance of the channel selection methods. The proposed ILFCS framework handles these errors by using $K$-means learning algorithm.

\subsection{Primary user activity model}

The performance of CRNs depends on the primary user activity over the channels. CR users can utilize the spectrum opportunistically only when the spectrum is not used by PUs. While CRs use an idle channel, primary user can arrive at any time; consequently, CRs have to end their communications and leave the current channel. Modeling the PUs' activity helps CR users to learn the history of primary user's spectrum utilization, and thus, they can predict their future state. In such a way, CR users can select the best available spectrum bands for their transmission. Therefore, the estimation of primary radio activity has a vital role in channel selection decision. We used the PU activity based on Poisson modeling with exponentially distributed inter-arrivals because it has been widely used in the literature.

The PU traffic is modeled as a two-state ON-OFF process. In this approach, each user arrival is independent, and it is assumed that the PU transmission follows the Poisson arrival process. Therefore, the length of $\mathrm{ON}$ and OFF periods are exponentially distributed. The period used by PUs is represented by an ON state while an OFF state represents the vacant periods. Such an ON/OFF PU activity model captures the time period in which the channel can be used by CRs without bringing out any harmful interference to PU nodes [12, 36].

\section{The proposed ILFCS framework}

As illustrated in Fig. 1, the proposed channel selection framework consists of four modules: (1) channel sensing module, (2) spectrum characterization module, (3) spectrum selection module, and (4) CR reconfiguration module. In the first module, each CR node senses the available channels using the spectrum sensing technique that is implemented in the physical layer. After that, the available channels pass to the second module that is implemented in MAC layer.

In the second module, the available spectrum channels are classified into four classes, (correct OFF, correct $\mathrm{ON}, \mathrm{FA}$, and $\mathrm{MD}$ ) based on the $K$-means unsupervised learning technique. This classification helps the CR to select true idle channels in an intelligent way and to avoid the channels with sensing errors (MD and $\mathrm{ON}$ ). This in turn leads to minimizing the spectrum sensing errors in the upper layer and subsequently enhances the spectrum usage level and reduces collision with PUs. The available channels (correct OFF and MD) pass to the spectrum selection module. In the third module, the channel with higher weight value is selected. This channel is associated with three parameters, PU utilization, channel capacity, and number of CRs on each channel, based on fuzzybased system. Consequently, the proposed framework has a cross layer scheme. Each layer has distinct role. After the best channel is selected, the QoS parameters will 


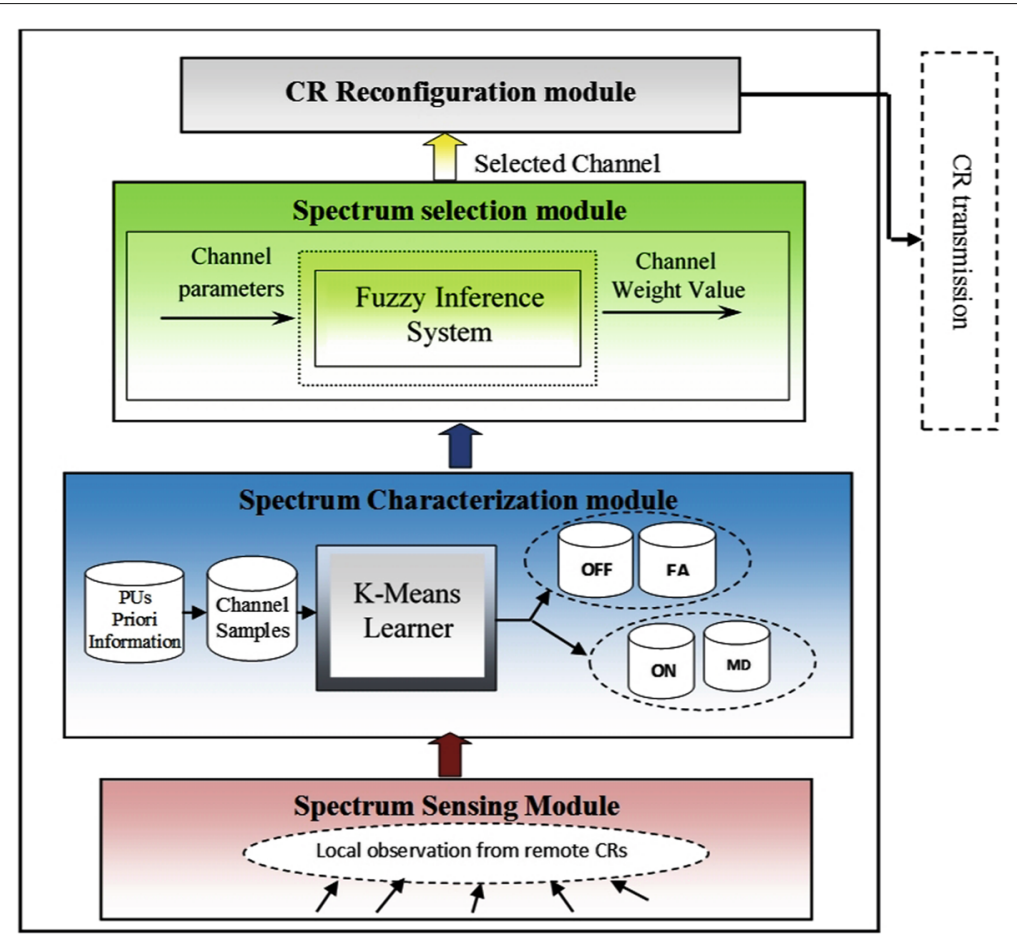

Fig. 1 ILFCS channel selection framework. The proposed channel selection framework modules is illustrated

be optimized in the fourth module. At the end, the CR makes a transmission using the selected channel and QoS parameters.

The selection of the appropriate channels for transmission in the proposed ILFCS is based on the channels that have low primary radio node utilization, less congestion with CR users and higher capacity. Channel parameters are fed into the fuzzy inference system in order to compute the channel weight value. Finally, the channel with the highest weight value is selected for transmitting the packet and the remainder channels are stored in a descending order to be kept as a backup for the selected channels. In the following subsections, the operation of $K$-means learner and the fuzzy channel selector are introduced in details. The contributions in this work focus on spectrum characterization and spectrum selection modules. In spectrum characterization module, $K$-means learning algorithm is proposed to handle the problem of sensing errors from the spectrum sensing technique. While in spectrum selection module, FIS is proposed to select the best channel.

\section{1 $K$-means unsupervised learning technique}

$K$-means is a type of neural network that belongs to the set of unsupervised learning techniques. One type of unsupervised learning problems is clustering (group similar data together). The $K$-means algorithm is a numerical clustering method where $K$ is a positive number initialized before the beginning of the algorithm in order to define the number of required groups. The $K$-means algorithm works in an iterative fashion. It starts by defining an initial set of clusters and the clusters are repeatedly updated until no more improvement is possible (or the number of iterations exceeds a specified limit)[37]. Figure 2 shows the steps of $K$-means clustering algorithm.

This paper utilizes the $K$-means algorithm to cluster the spectrum channels according to their states into four groups (correct OFF, correct ON, FA, and MD).This division is done based on the state of the channel. According to the sensing results collected by each CR, three features determine each channel state. These features are the state of sensing, the state of the corresponding interference due to the presence of the PU inside its sensing range (internal PU), and the state of the presence of the PU located outside (external PU). The proposed $K$-means composed of three inputs features and four outputs. These inputs are the three mentioned features of each channel, while the outputs cluster the spectrum channels according to their states into four groups (correct OFF, correct ON, FA, and $\mathrm{MD}$ ). Each channel state is expressed by one vector as shown in Table 1 [30]. The correct OFF cluster covers the samples that show the OFF sensing without any interference from internal or external PU expressed by the vector (000). However, the FA cluster contains the samples 


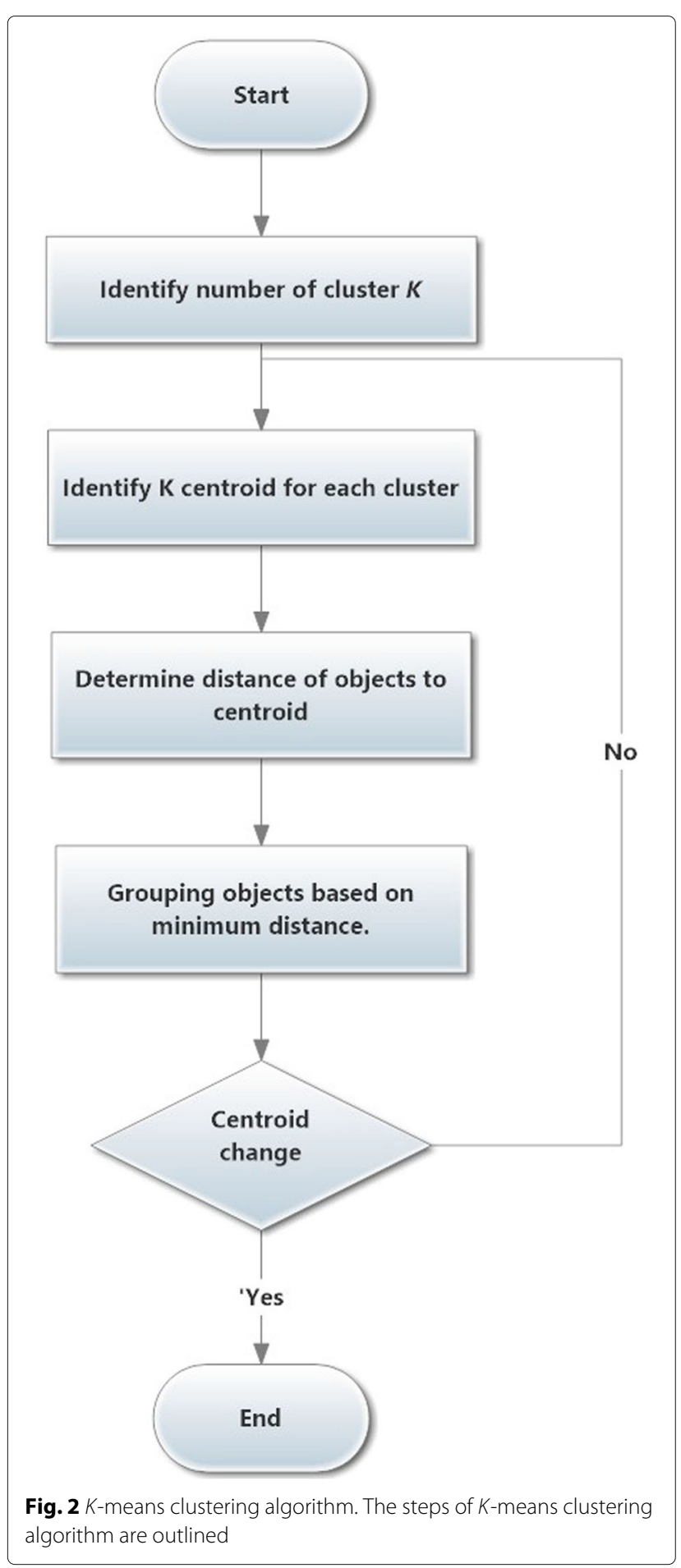

that indicate the erroneous sensing report caused when CR user discovers the channel ON at time $(t)$, but one $\mathrm{CR}$ neighbor discovers the channel OFF at time $(t)$ and completed a transmission on channel during $(t-\Delta t, t)$. The FA state is expressed by the vector (101). The correct $\mathrm{ON}$ cluster comprises the samples that indicate the
ON sensing without any possibility for external PU and is expressed by the vector [110]. The MD cluster covers the samples that indicate the off sensing while $C R$ neighbors discovers channel $\mathrm{ON}$ and is expressed by the vector [010].

Iteratively, the $K$-means learner receives a sample for each channel. The learning step is independently performed for each channel. After sufficient iterations, the $K$-means can predict the suitable cluster for next samples. For dynamic changes in real environment, the number of samples for channel $(k)$ may increase in a certain cluster resulting in changing the probability of channel state. After this classifier works, the output from $K$-means passes to the fuzzy inference model as depicted in Fig. 2, where only FA and correct OFF groups will send to the FIS.

\subsection{Fuzzy channel selection module}

The most important challenge to maximize the performance of a CRN is to minimize interference caused to PUs and among CRs. Thus, best channels with minimum PUs' activities, less congested with CR users, and high capacity should be selected. Output channels resulted from $K$-means clustering are classified by assigning a weight value to each channel. FIS system is used to calculate each channel weight value considering three parameters: primary user's utilization, cognitive user's number, and channel capacity.

\subsubsection{The fuzzy input parameters}

\subsubsection{A. Primary user's utilization}

Primary user channel utilization is the fraction of time in which channel $i$ is in on state, i.e., utilized by primary nodes. Channel utilization $u$ is calculated by Eq. 1 [36]. The most important requirement for CR networks is that CRs should not interfere with licensed PUs transmission. Therefore, the best channel is the one with low primary user's utilization rate.

$$
U_{i}=\frac{E\left[T_{O N}^{i}\right]}{E\left[T_{O N}^{i}\right]+E\left[T_{O F F}^{i}\right]}=\frac{\lambda_{y}}{\lambda_{x}+\lambda_{y}}
$$

Where, $E\left[T_{O N}^{i}\right]=\frac{1}{\lambda_{x}}, E\left[T_{O F F}^{i}\right]=\frac{1}{\lambda_{y}}, \lambda_{x}, \lambda_{y}$ are rate parameters for exponential distribution, and $E\left[T_{O N}^{i}\right], E\left[T_{O F F}^{i}\right]$ are the mean exponential distribution.The values of $\lambda_{x} a n d \lambda_{y}$ can be easily measured by $C R$ nodes by collecting the historical samples of channel state transitions. Values of $\lambda_{x}$ and $\lambda_{y}$ recorded in [36] are used to calculate channel utilization as shown in Table 2.

\subsubsection{B. Cognitive user's number}

Good channel selection strategy chooses the channel with lower number of $\mathrm{CR}$ neighbors using the channel. Lower number of CR reduces interference among CRs, which increases transmission rate, resource utilization, 
Table 1 Samples space for channel states

\begin{tabular}{|c|c|c|c|c|c|}
\hline Sample rule & $\begin{array}{l}\text { Current } \\
\text { sensing }\end{array}$ & $\begin{array}{l}\text { Interference with } \\
\text { internal PU }\end{array}$ & $\begin{array}{l}\text { Interference with } \\
\text { external PU }\end{array}$ & $\begin{array}{l}\text { Generated } \\
\text { sample }\end{array}$ & $\begin{array}{l}\text { Channel state } \\
\text { description }\end{array}$ \\
\hline $\begin{array}{l}\text { Either CR user or one CR neighbor } \\
\text { discovers the channel OFF at time } \\
(t) \text { and completed a transmission } \\
\text { on channel during }[t-\Delta t, t]) \text {. }\end{array}$ & 0 & 0 & 0 & {$\left[\begin{array}{lll}0 & 0 & 0\end{array}\right]$} & OFF \\
\hline $\begin{array}{l}\text { CR user discovers the channel OFF } \\
\text { at time }(t) \text {, but failed to transmit on } \\
\text { channel during }(t-\Delta t, t) \text { or most } \\
\text { CR neighbors discovers the } \\
\text { channel ON at time }(t-\Delta t) \text { and } t \text {. }\end{array}$ & 1 & 1 & 0 & {$\left[\begin{array}{lll}0 & 1 & 0\end{array}\right]$} & $\mathrm{MD}$ \\
\hline $\begin{array}{l}\text { CR user discovers the channel ON } \\
\text { at time }(t) \text {, and most CR neighbors } \\
\text { discovers the channel ON at time } \\
(t-\Delta t) \text { and } t \text {. }\end{array}$ & 1 & 1 & 0 & {$\left[\begin{array}{lll}1 & 1 & 0\end{array}\right]$} & ON \\
\hline $\begin{array}{l}\text { CR user discovers the channel ON } \\
\text { at time }(t) \text {, but one CR neighbor } \\
\text { discovers the channel OFF at time } \\
(t) \text { and completed a transmission } \\
\text { on channel during }(t-\Delta t, t) \text {. }\end{array}$ & 1 & 0 & 1 & {$\left[\begin{array}{lll}1 & 0 & 0\end{array}\right]$} & FA \\
\hline
\end{tabular}

and throughput, and at the same time, it decreases packet loss ratio and packet delay. In order to determine the number of CR users, each CR node discovers their neighbors by using a common control channel (CCC) mechanism.

\subsubsection{Channel capacity}

Channel capacity indicates users data rate per each $\mathrm{HZ}$ of the spectrum band used. The expected normalized capacity of a user $k$ in spectrum band $i$ is calculated using Eq. 2 [12].

$$
C_{i}^{C R}(k)=E\left[C_{i}(k)\right]=\frac{T_{i}^{o f f}}{T_{i}^{o f f}+\tau} \cdot \gamma_{i} \cdot c_{i}(k)
$$

where $C_{i}(k)$ represents spectrum capacity, $c_{i}(k)$ is the normalized channel capacity of spectrum band $i$ in $\mathrm{bits} / \mathrm{sec} / \mathrm{Hz}, \tau$ is the spectrum switching delay, $\gamma_{i}$ is the spectrum sensing efficiency, and $T_{i}^{\text {off }}$ represents the expected transmission time without switching in spectrum band.

\subsubsection{Fuzzy logic inference system}

The fuzzy inference system employs fuzzy logic concepts to perform tasks such as decision making. The block diagram of the fuzzy inference system is shown in Fig. 3. There are four basic steps summarize the operation of the fuzzy logic algorithms [38, 39]:

Table 2 Wireless channel parameters

\begin{tabular}{lllllllllll}
\hline & Ch 1 & Ch 2 & Ch 3 & Ch 4 & Ch 5 & Ch 6 & Ch 7 & Ch 8 & Ch9 & Ch 10 \\
\hline$\lambda_{x}$ & 1.25 & 0.4 & 1 & 0.4 & 0.5 & 2 & 1 & 0.18 & 0.5 & 0.67 \\
$\lambda_{y}$ & 0.67 & 2 & 1 & 0.33 & 1 & 0.29 & 0.25 & 2 & 1.33 & 0.5 \\
$u^{i}$ & 0.35 & 0.83 & 0.5 & 0.45 & 0.67 & 0.13 & 0.2 & 0.92 & 0.73 & 0.43 \\
\hline
\end{tabular}

- Fuzzification system inputs (crisp numbers) are changed into fuzzy sets by applying a fuzzification function.

- Rule evaluation stores IF-THEN rules written by a professional designer in the related field.

- Inference engine simulates the human reasoning process by making fuzzy inference on the inputs and IF-THEN rules.

- Defuzzification converts the fuzzy outputs obtained by the inference engine into a crisp value.

\subsubsection{Fuzzy logic algorithm description}

In the proposed ILFTS , Mamdani's method fuzzy inference technique [40] with three inputs and one output is used. Fuzzy system used for our proposed model is shown in Fig. 4. In fuzzy inference system, the input and output variables should be represented as membership function [41].

In fuzzifer, crisp (actual) values are converted into membership functions as shown in Fig. 5. There are different forms of membership functions such as: triangular, trapezoidal, Gaussian, and singleton. In our case, triangular membership functions are used. The following equation describes triangular function defined by a lower limit $a$, an upper limit $b$, and a value:

$$
\mu_{A}(x)= \begin{cases}0 & x \leq a \\ \frac{x-a}{m-a} & a<x \leq m \\ \frac{b-x}{b-m} & m<x \leq b \\ 0 & b \geq x\end{cases}
$$

$m$ where $a<m<b$. Fuzzified values are processed by if then statements according to a set of predefined rules written by a professional designer in the related field. In our case, we have used 27 IF-THEN-based rules (Table 3). 


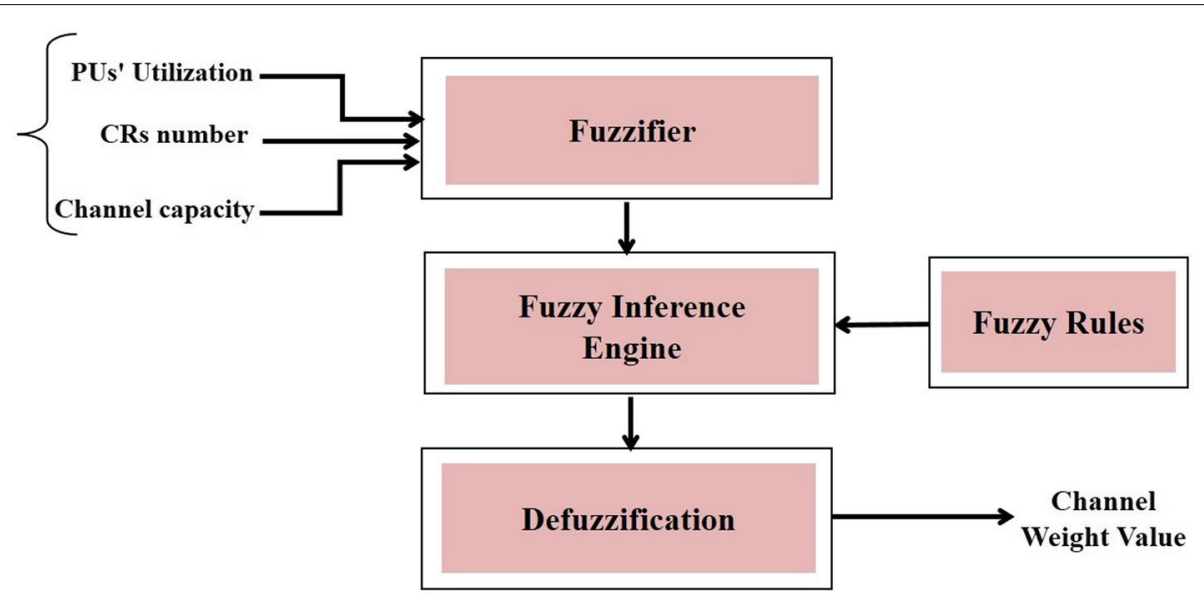

Fig. 3 Fuzzy inference system. The block diagram of the fuzzy inference system are shown

The fuzzy operator used here is the AND operator. For example, if utilization is low and CR users' number is low and channel capacity is high then the channel weight value is high. This means that this channel is a most trusted one. The outputs obtained for each rule are combined to obtain a final result using the maximum operator. Finally, the obtained fuzzy value is defuzzified to obtain a final crisp value. Center of gravity defuzzification method is used.

It is worth mentioning that the proposed algorithm consists of two parts, (1) $K$-means and (2) fuzzy-based system. In time complexity analysis of $K$-means, the time spent in the learning phase is ignored since it is carried out "off-line". The corresponding time cost of its working phase is $O(I J)^{2}$ because the $K$-means network is fully connected so that every input, $i=1,2, \ldots, j$ is connected to all output nodes, $j=1,2, \ldots, j$ (here, 3 inputs and 4 outputs are used). However, such time cost is not crucial because the numbers of input and output $K$-means nodes are always limited to the channel status (either $\mathrm{ON}$ or OFF). On the other hand, depicts the time complexity of segregated selection is $O(F I D)$, where, $F$ is the time spent in (Fuzzification) process, $I$ is the time spent in (Fuzzy Inference Engine), and $D$ is the number of candidate channels and $k$ is the heap lists. Consequently, the overall time cost of the proposed algorithm will be $O(I . J)^{2}+O(F I D)$ (Fig. 3).

\section{Results and discussion}

In this section, the performance of the proposed selection framework (ILFCS) through extensive simulations will be analyzed. In the following part, simulation results for the proposed framework will be introduced by the network simulator (NS2). The output of FIS, which is the channel weight value, is applied to the NS2 simulation for evaluation. The work flow between the NS2 and the MATLAB is demonstrated in Fig. 6.

\subsection{Implementation setup}

On NS2 simulator, the CR node and the required layers for network functionality were developed. Cognitive radio

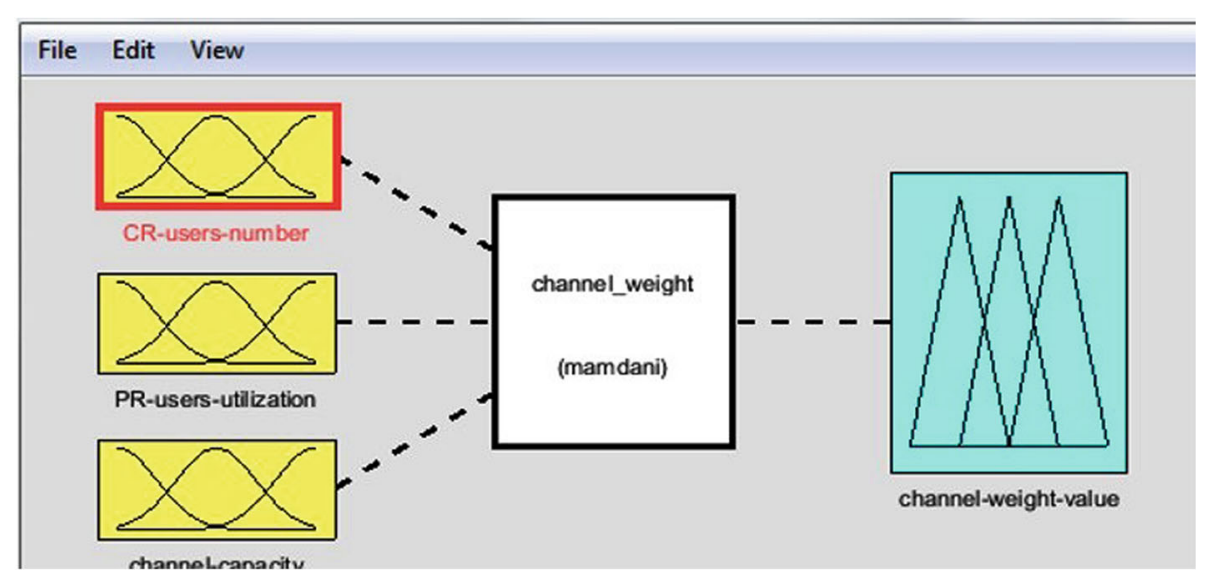

Fig. 4 Fuzzy system for the proposed model. The inference techniques and the fuzzy system used for the proposed ILFCS strategy are demonstrated 
cognitive network (CRCN) patch of NS2 is used. The CRCN patch has three building blocks that support cognitive radio functionalities in NS2. These blocks are cognitive radio physical layer, cognitive radio MAC layer, and cognitive radio network layer [42]. The CR network layer is accountable for keeping and maintaining the neighbor list. It also decides on the channel selection. This decision is passed to the MAC layer which then handles switching to the selected channel. The CR physical layer is responsible for sensing some information like all the available spectrum bands, transmission power, propagation model, and SINR/SNR physical model. This CRCN patch of NS2 does not model the activity of the PU nodes. Here, the CRCN patch of NS-2 is modified to include the used PU activity model. The PU activity block is responsible for generating and keeping track of PU activities in each spectrum band (spectrum utilization), that is, the sequence of ON and OFF periods by primary nodes over the simulation time. These ON and OFF periods can be modeled as continuous time, alternating ON/OFF Markov Renewal Process (MRP) [42]. The ON (busy) state indicates that the primary node occupies the channel. While, the OFF (idle) state means that the channel is unoccupied by the primary node. The channels ON and OFF periods are considered exponentially distributed. The rate parameters of exponential distribution $\lambda_{x}, \lambda_{y}$ listed in Table 2 which was measured in [36] are provided as inputs in simulation.

A simple MAC protocol (Maccon.cc), which is available with the CRCN patch of ns-2, will be considered in the study. This MAC protocol is a multiple-channel, collision- and contention-based MAC protocol. In the original state, the Maccon.cc MAC protocol randomly selects a channel from the predefined set of channels, and the channel selection decision occurs at the MAC layer. In the proposed ILFCS, channel selection is done at the network layer. Thus, we have modified this MAC protocol to provide the ability for the network layer to make the channel selection decision. Based on the channel selection strategy, the network layer takes the channel selection decision. This decision is encapsulated in the network layer packet header and passed to the MAC layer, which then switches to the channel based on the channel selection decision that is provided by the network layer [42].

\subsection{Performance metrics}

The efficiency of ILFCS compared to other strategies is verified using NS2 simulator concerning the following performance metrics:

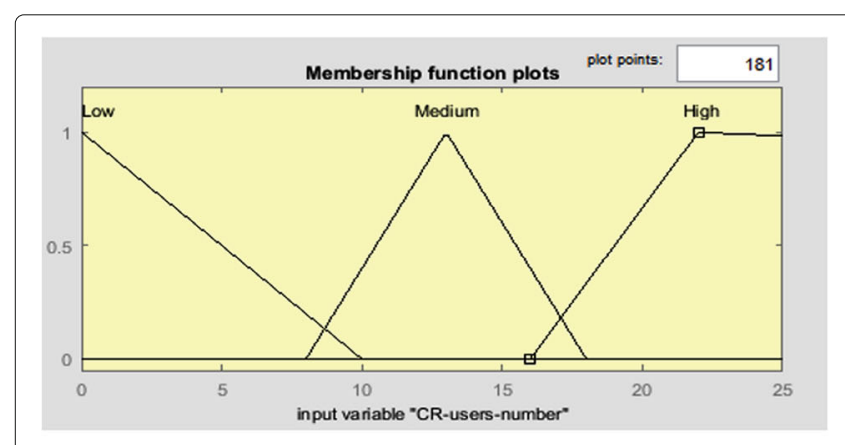

a

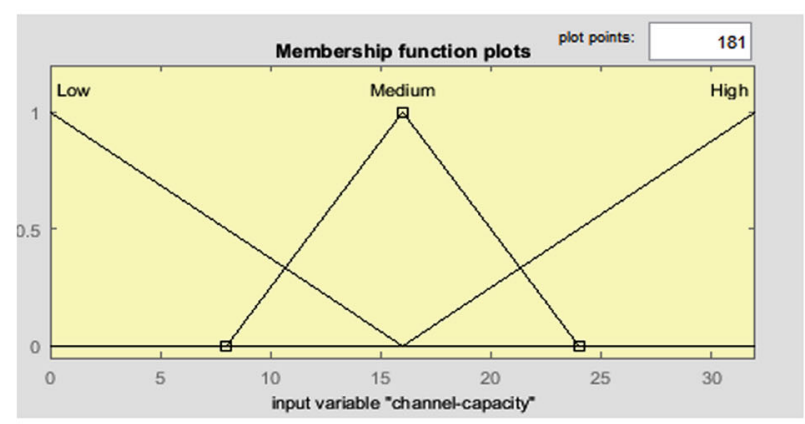

C

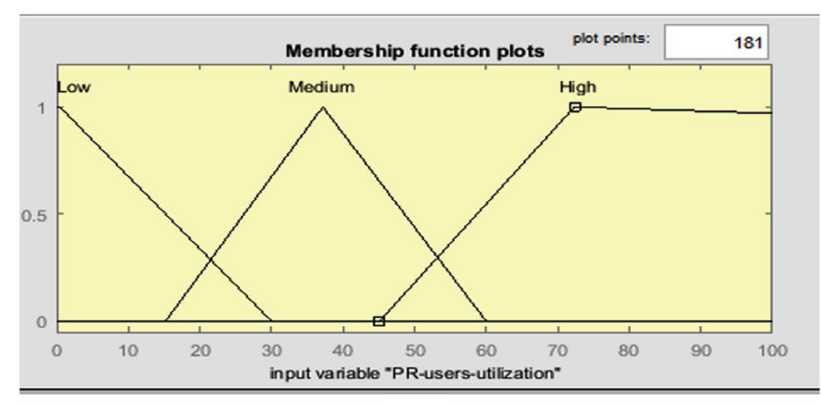

b

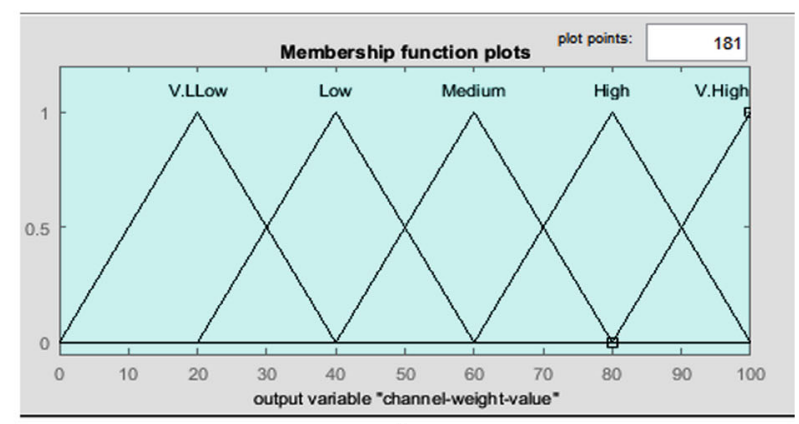

d

Fig. 5 Fuzzy membership sets of the input and output variables: a CR users' number. b PUs' utilization. c Channel capacity. d Fuzzy set for output weight value. The fuzzy membership sets of the input and output variables are shown 
Table 3 Fuzzy base rule set

\begin{tabular}{|c|c|c|c|}
\hline PU utilization & CR users umbers & Channel capacity & Output weight value \\
\hline High & High & High & Low \\
\hline High & High & Medium & Low \\
\hline High & High & Low & Very low \\
\hline High & Medium & High & Low \\
\hline High & Medium & Medium & Low \\
\hline High & Medium & Low & Very low \\
\hline High & Low & High & Low \\
\hline High & Low & Medium & Low \\
\hline High & Low & Low & Very low \\
\hline Medium & High & High & Medium \\
\hline Medium & High & Medium & Low \\
\hline Medium & High & Low & Very low \\
\hline Medium & Medium & High & Medium \\
\hline Medium & Medium & Medium & Medium \\
\hline Medium & Medium & Low & Low \\
\hline Medium & Low & High & High \\
\hline Medium & Low & Medium & Medium \\
\hline Medium & Low & Low & Low \\
\hline Low & High & High & Medium \\
\hline Low & High & Medium & Low \\
\hline Low & High & Low & Low \\
\hline Low & Low & High & Very high \\
\hline Low & Low & Medium & Very high \\
\hline Low & Low & Low & Very low \\
\hline Low & Medium & Low & Low \\
\hline Low & Medium & Medium & Low \\
\hline Low & Medium & High & High \\
\hline
\end{tabular}

- Spectrum opportunity utilization. It is the ratio of the total time of successful CR users' transmission to the total PUs' idle time.

- Average interference ratio. It is defined as the ratio of the PU channel occupation time to total times the channel selection decision times.

- Average throughput. It is defined as the average number of packets successfully received by CR destination nodes during the total simulation time.

- Packet delivery ratio. It is the average number of received packets to the number of sent packets.

- End-to-end delay. It is the total time taken for a packet to reach the destination

\subsection{Simulation environment}

Before presenting NS2 simulation results, we present basic assumptions and the simulation parameters used.

\subsubsection{Assumptions}

We consider source destination pairs in single-hop context. Therefore, in this communication scheme, no routing is required; thus, neither routing tables nor end-to-end paths are maintained. The time spent of $K$-means algorithm in the learning phase is ignored since it is carried out "off-line". The corresponding time cost of its working phase is small.

CR nodes use a CSMA/CA-based medium access protocol. Contentions among CRs are resolved using carrier sensing and back-off mechanism. Both medium access control and PU activity models are included in CR MAC layer.

Each CR has a single radio transceiver that can be tuned to various frequencies licensed to the primary network. Sensing and transmission are performed sequentially due to the single radio restriction.

The rate parameters of exponential distribution $\lambda_{x} a n d \lambda_{y}$ are listed in Table 2 which are adopted from [36].

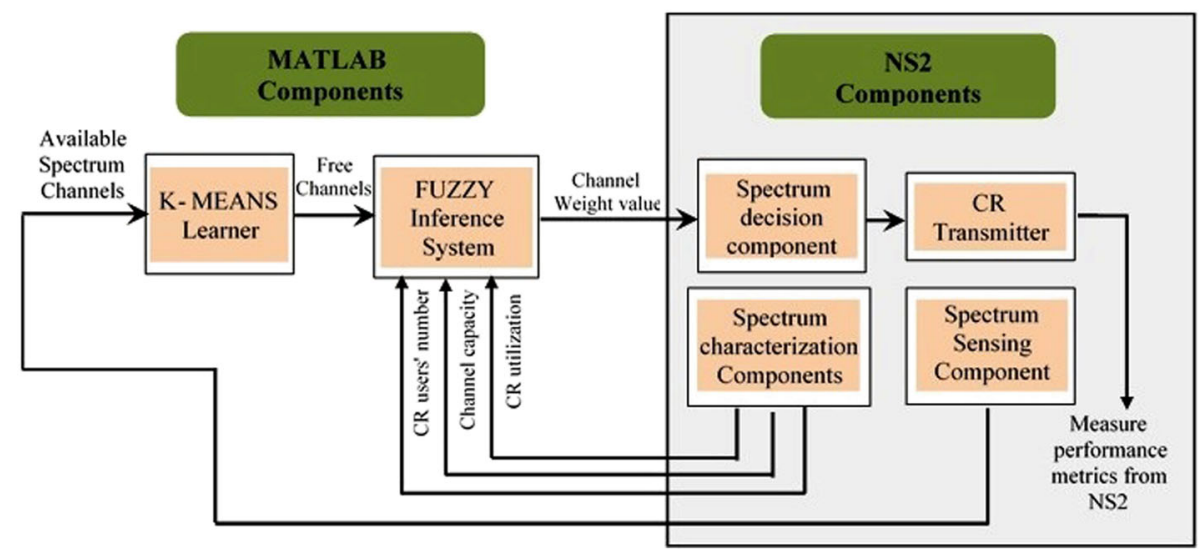

Fig. 6 The work flow between MATLAB and NS2 
These values are provided as inputs in the simulation experiments which evaluate the impact of varying $C R$ users density and varying the number of channels on the performance of the selection strategies.

\subsubsection{Simulation parameters}

As illustrated in Table 5, CR nodes are randomly deployed within a square area of A2 $=700 \times 700 \mathrm{~m} 2$. Simulations run for $600 \mathrm{~s}$. Total 600 packets were sent. Each packet is sent by a randomly selected node. The packet size is set to 512 bytes. The transmission range of CR nodes is set to $250 \mathrm{~m}$. Simulation parameters used are listed in Table 4.

\subsection{Performance validation and discussion}

A performance comparison for ILFCS, best-fit channel selection (BFC), GA-based selection (GA), Intelligent Channel Selection Scheme a Self Organized Map Followed by Simple Segregation (ICSSSS), and longest idle time channel selection (LITC) has been evaluated concerning different numbers of CR nodes, different number of channels, and different PU activity patterns.

\subsubsection{Impact of varying CR users density on the channel selection strategies performance}

The impact of the growth of CR users traffic demand on each scheme is studied considering the mentioned performance metrics. The number of available channels at each CR users is set to 10 . The behavior of PUs activities are not identical for all the available channels. It varies from channel to channel. The number of CR users in the network varies from 10 to 250 . Simulation results show that the ILFCS achieves its minimum enhancement in the average interference ratio compared with ICSSSS when

Table 4 The simulation parameters

\begin{tabular}{lll}
\hline Simulation object & Parameter & Value \\
\hline Network node & Transmission media & Wireless channel \\
& Propagation model & $\begin{array}{l}\text { Free space model, two-ray } \\
\text { ground model, } \\
\text { Fading/shadowing model }\end{array}$ \\
& Network interface & WirelessPhy \\
& Number of interfaces & Single transceiver \\
& MAC & 802.11 \\
& Antenna & Omni Antenna \\
& Interface queue type & DropTail/Priqueue \\
& Packet size & 512 bytes \\
& Transmission range & 250 meters \\
CRAHN network & Number of CR users & $10-250$ \\
& K-means inputs & 3 \\
\hline
\end{tabular}

the number of CR nodes are 25 and 250. As it is shown in Fig. 7, the ratio is decreased by around $5 \%$ compared with ICSSSS. ILFCS achieves the maximum average interference ratio compared with LITC when the number of CR nodes are 150 and 175 as the ratio is decreased by $70 \%$. Moreover, it is noticed that ILFCS outperforms LITC, GA, $\mathrm{BFC}$, and ICSSSS where it reduces the average interference ratio by $60 \%, 40 \%, 32 \%$, and $7 \%$ compared to LITC, GA, BFC, and ICSSSS respectively.

The experimental results depicted in Fig. 7 show that when the number of CRs increased, the ratio of interference is increased in all schemes. This is due to the increase of CRs' density of the network which increase the contention and interference ration between CRs. In addition, the inaccurate prediction of PUs traffic leads the CR user to select the wrong channel. Accordingly, PU disconnect the transmission of the CR. This increase the CR users channel switching rate. The ILFCS outperforms all related scheme because the prediction of PU is estimated in an intelligent way with removing the sensing errors from the list of available channel.

As shown in Fig. 8, concerning average throughput, the ILFCS achieves its minimum enhancement compared with ICSSSS when number of CR nodes is 225. As it is shown, the ratio is increased by around $5.3 \%$ compared with ICSSSS, while achives its maximum average throughput compared with LITC when number of CR nodes is 75 . As it is shown, the ratio is increased by $48.8 \%$ compared with LITC. It can be realized that ILFCS outperforms LITC, GA, BFC, and ICSSSS where the results show that average throughput is maximized at different numbers of CR nodes by $47.9 \%, 29.8 \%, 18.3 \%$, and $8.6 \%$ respectively. The average throughput is more related to the ratio of the

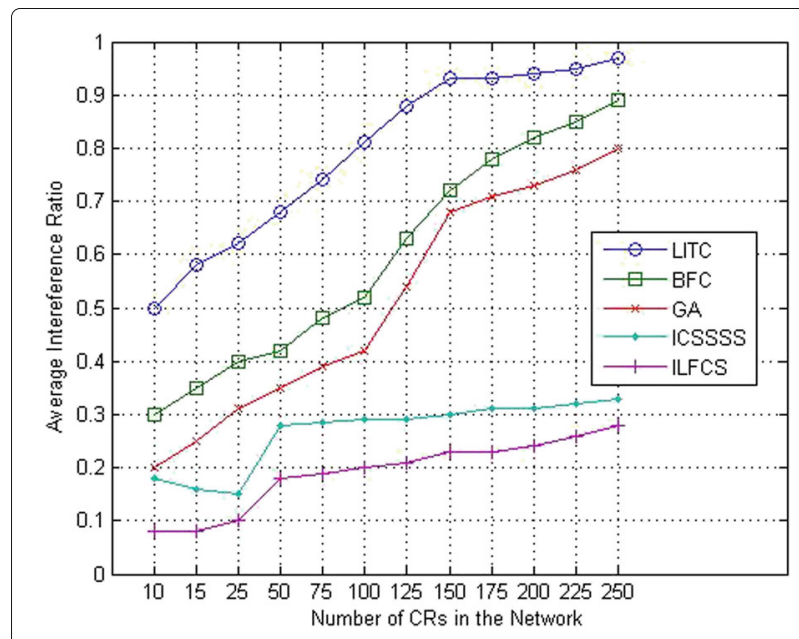

Fig. 7 Average interference ratio undervariant network density. The results of diverse simulation experiments to measure the average interference ratio at different numbers of CR nodes for the BFC, LITC, GA, ICSSSS, and the ILFCS are illustrated 


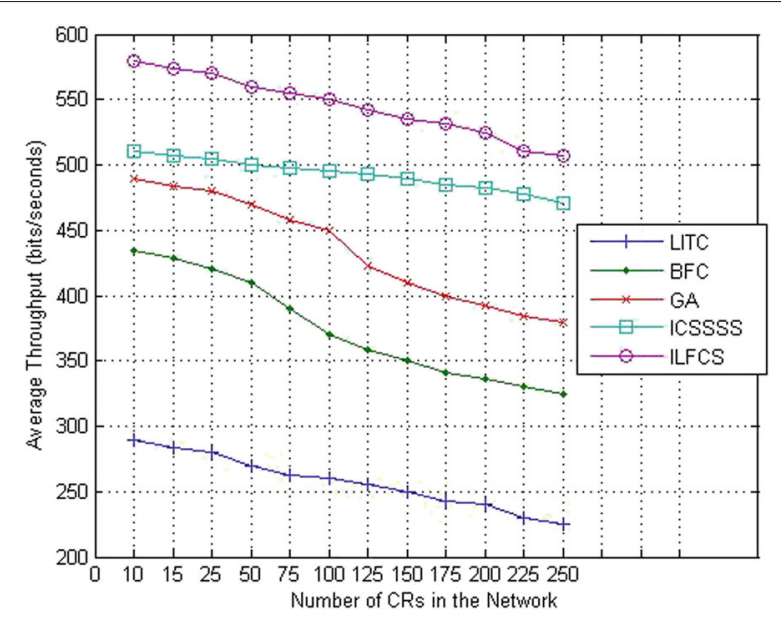

Fig. 8 Average throughput under different network densities. The results of diverse simulation experiments to measure the average throughput at different numbers of CR nodes for the BFC, LITC, GA, ICSSSS, and the ILFCS are illustrated

total packets received in the network and the ratio of interference. When the number of CRs successfully completed its transmission, this leads to the increase of the total number of successfully received packets. Consequently, the average throughput increases.

As depicted in Fig. 9, in all schemes, when the number of CRs increased, the packet delivery ratio decreased, the main reason for that, with increasing in the density of CRs in the networks, contention between them are high and the ratio of interference increased, but different from scheme to scheme. In addition, the ILFCS outperforms other scheme because the ratio of interference is

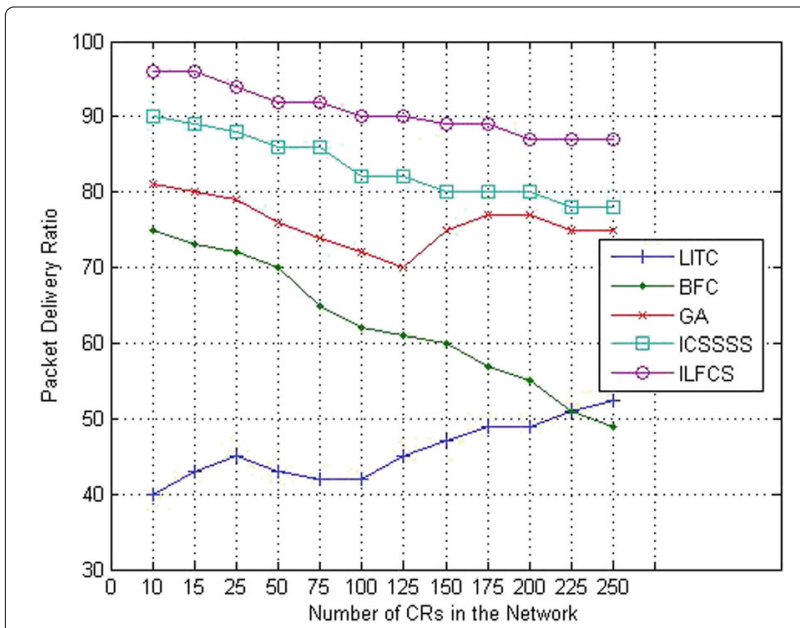

Fig. 9 Packet delivery ratio under different network density. The results of diverse simulation experiments to measure the Packet delivery ratio at different numbers of CR nodes for the BFC, LITC, GA, ICSSSS, and the ILFCS are illustrated small. This leads to the increase in the packet delivery ratio which in turn increases the average throughput over the network in specific time.

The figure shows the packet delivery ratio at different numbers of CR nodes for the LITC, BFC, GA, ILFCS, and ICSSSS. It can be realized that ILFCS outperforms ILTC, BFC, GA, and ICSSSS by achieving maximum packet delivery ratio at different network densities by $45 \%, 28.3 \%$, $14.8 \%$, and $7.5 \%$ respectively.

For all scheme, when the number of CRs increased, the packet delivery ratio decreased. As with the increase in number of the CRs in the network the competition for the unoccupied channels becomes more compelling. Consequently, the average throughput per CR decreases in all schemes. Moreover, selecting the best channel in an intelligent process by using $K$-means and FIS techniques leads to decrease the interference between CRs-CRs and CRs-PUs. Hence, the packets sent over the network will transmitted successfully without any interrupt with PUs connections.

Figure 10 illustrates the results of measuring the spectrum opportunity utilization at different numbers of CR nodes for the LITC, BFC, GA, ICSSSS, and ILFCS. ILFCS achieves its minimum enhancement in the spectrum opportunity utilization compared with ICSSSS when number of CR nodes is 150 . As it is shown, the ratio is increased by $3 \%$ compared with ICSSSS, while the maximum average interference ratio is achieved compared with LITC when number of CR nodes is 75 where the ratio is increased by $63 \%$ compared with LITC. It can be realized that ILFCS always outperforms LITC, BFC, GA, and ICSSSS. The opportunity utilization is maximized at different number of CR nodes by $57.8 \%, 41.4 \%$,

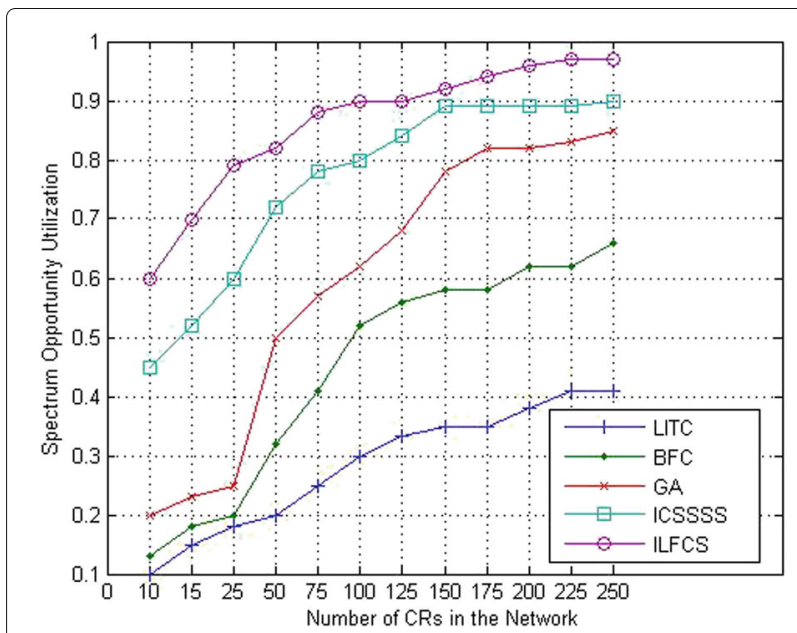

Fig. 10 Spectrum opportunity utilization under different network density. The results of diverse simulation experiments to spectrum opportunity utilization at different numbers of CR nodes for the BFC, LITC, GA, ICSSSS, and the ILFCS are illustrated 
26.7\%, and 9.8\% compared to LITC, GA, BFC, and ICSSSS respectively.

Spectrum utilization is more related to packet delivery and interference ratio. Therefore, the spectrum utilization ratio increases when interference ratio decreased and packets delivery ratio increases and vice versa. ILFCS outperforms other schemes because the assistance of the $K$-means and FIS in selecting the channel so that guarantees that the channel not utilized by PU. Therefore, the interference between users decreased that leads to increasing in spectrum utilization in the proposed framework.

Finally, Fig. 11 illustrates the results of measuring the end-to-end delay at different numbers of CR nodes for the LITC, BFC, GA, ICSSSS, and ILFCS. ILFCS achieved its minimum enhancement in end-to-end delay compared with ICSSSS at 150 CR nodes where the ratio is decreased by around $0.8 \%$ compared with ICSSSS, while the maximum enhancement compared with LITC occurred at 250 CR nodes at which the ratio is decreased by $90.6 \%$ compared with LITC. It is noticed that ILFCS outperforms LITC, BFC and GA and ICSSSS. The result shows that the end-to-end delay is decreased by $88.7 \%, 84.4 \%, 77.8 \%$, and $28.3 \%$ compared to LITC, BFC, GA, and ICSSSS respectively. Actually, ignored sensing error in selecting the best channel leads to a critical decrease in the interference ratio. Therefore, the hopping rate for $\mathrm{CR}$ from channel to another channel is high. So the switching time for discovering new available channel takes a lot time which leads to increasing the average end-to-end delay over the network.

In ILFCS, sensing errors are handled by $K$-means algorithm to remove ON state channels and MD channels. This leads to select the best channel in an intelligent way.
Consequently, CRs are allowed to transmit their packets without any interference with PUs or switching from channel to other which in turn reduces the average delay over the entire network.

\subsubsection{Impact of varying the number of channels on the channel selection strategies performance}

In this set of simulations, the effect of different number of channels on the performance of each scheme is examined considering the mentioned performance metrics. The number of $C R$ users is 50 nodes. The behavior of PUs' activities are not identical for all the available channels. It varies from channel to channel. The number of available channels at each $C R$ varies from 3 to 15 .

Figure 12 shows the results of the average interference ratio at different numbers of available channels for LITC, GA, BFC, ICSSSS, and ILFCS. On average, it is seen that, ILFCS minimizes the interference ratio by $56.8 \%, 29.9 \%$, $24.9 \%$, and $4.4 \%$ compared to LITC, GA, BFC, and ICSSSS respectively.

Figure 13 demonstrates the results of the average throughput at different numbers of available channels for GA, BFC, LITC, ICSSSS, and ILFCS. On average, it is noticed that the average throughput is increased by $49.6 \%$, $29.3 \% 24.2 \%$, and $12.7 \%$ compared to LITC, GA, BFC, and ICSSSS respectively.

Figure 14 shows the result of the packet delivery ratio at different numbers of available channels for the LITC, BFC, GA, ICSSSS, and ILFCS. The results demonstrate that ILFCS can enhance the packet delivery ratio by $46.8 \%$, $29.3 \%, 13.7 \%$, and $6.1 \%$ compared to LITC, BFC, GA, and ICSSSS respectively.

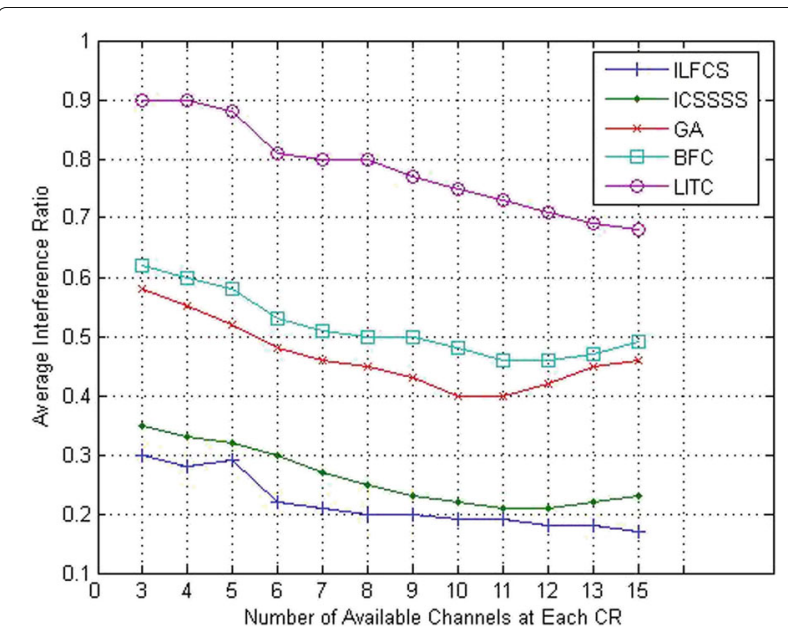

Fig. 12 Average interference ratio under different number of available channels. The results of different simulation experiments to measure average interference ratio at different numbers of available channels for the BFC, LITC, GA, ICSSSS, and the ILFCS are shown 


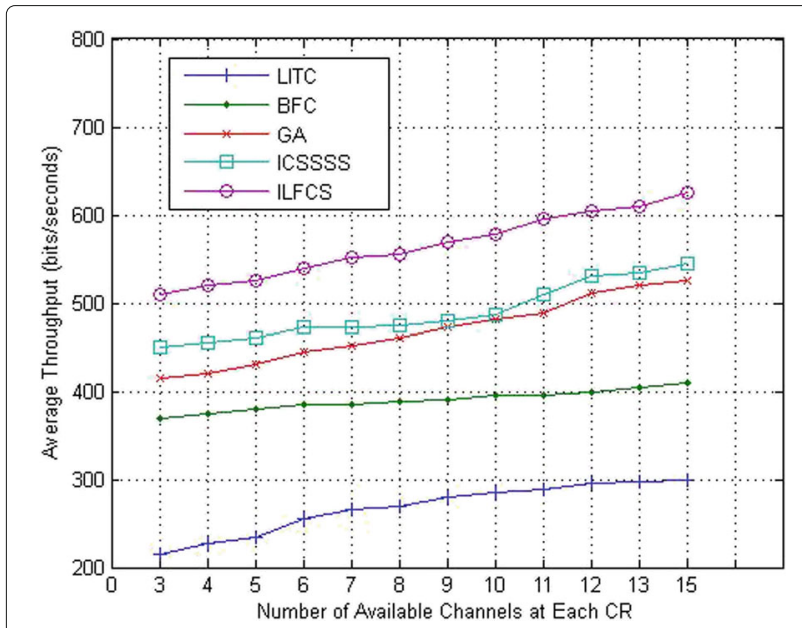

Fig. 13 Average throughput under different number of available channels. The results of different simulation experiments to measure average throughput at different numbers of available channels for the BFC, LITC, GA, ICSSSS, and the ILFCS are shown
Figure 15 demonstrates the result of spectrum opportunity utilization for ILFCS, LITC, BFC, GA, and ICSSSS at different numbers of channels. It can be concluded that ILFCS scheme always outperforms LITC, BFC, GA, and ICSSSS. Spectrum opportunity utilization is increased by $83.2 \%, 31.7 \%, 27.7 \%$, and $6.7 \%$ compared to LITC, GA, BFC, and ICSSSS respectively.

The results of measuring the end-to-end delay at different numbers of channels for the LITC, GA, BFC, ICSSSS, and ILFCS are illustrated in Fig. 16. On the average, the end-to-end delay of ILFCS is decreased by $75.6 \%$,

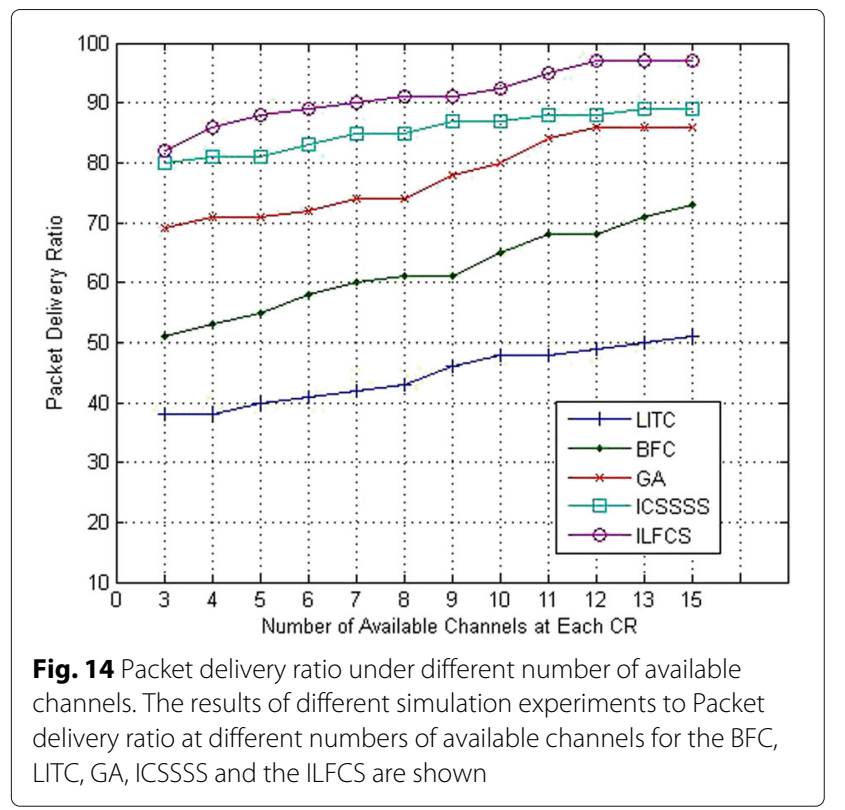

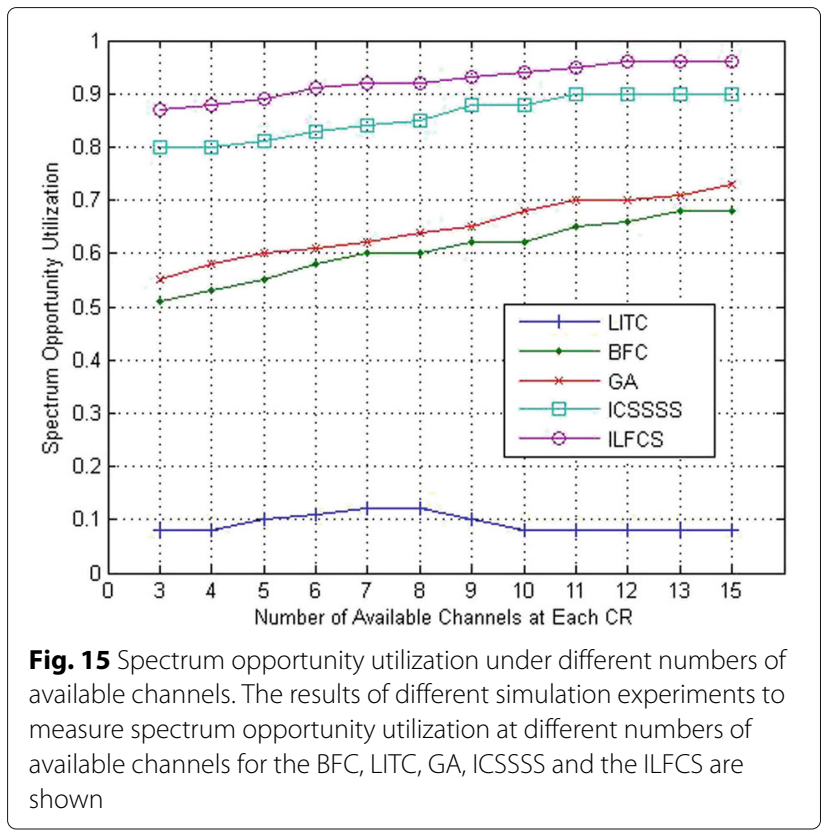

71.3\%, $60.8 \%$, and $50.8 \%$ compared to LITC, BFC, GA, and ICSSSS respectively.

It can be concluded that the network performance is directly proportional to the number of channel. The low number of channels decreases the opportunity for CR users to find free channels for transmission. In addition, the ILFCS outperforms all other schemes because the proposed framework guarantee that the best channel is selected in an intelligent way and ensure that not include sensing errors. Hence, the average interference ratio between CRs-PUs decreased which leads to more

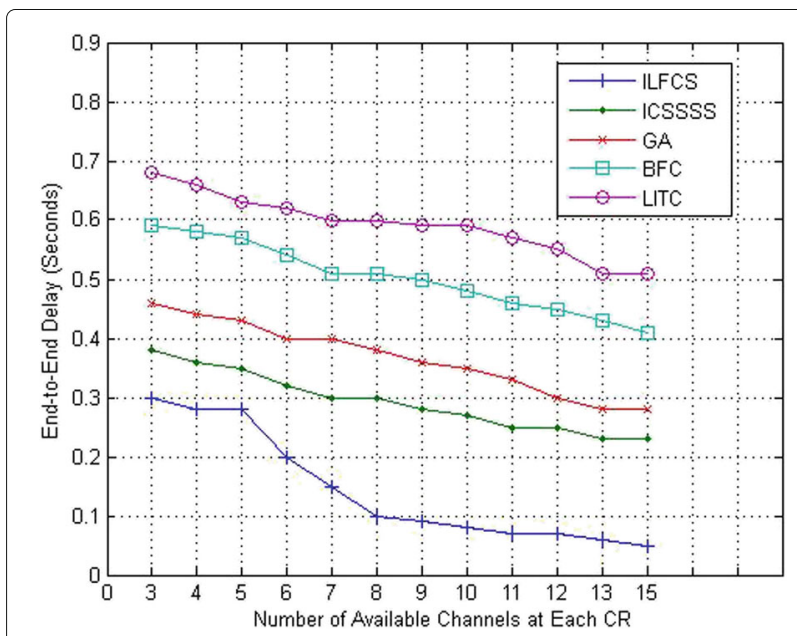

Fig. 16 End-to-end delay under different number of available channels. The results of different simulation experiments to measure End-to-end delay at different numbers of available channels for the BFC, LITC, GA, ICSSSS and the ILFCS are shown 
packet transmission successfully over the network; consequently, the average throughput increased.

\subsubsection{Impact of varying PU activity pattern on the channel selection strategies}

In this section, we study the different PU activity patterns' impact on the proposed channel selection strategy ILFCS and each of GA, LITC, BFC, and ICSSSS. We consider then three different PU activity patterns [36], described as follows:

- Long-term PR activity: In long-term PR activity, the channel has long ON and long OFF periods.

Scenarios where primary radio nodes subscribed to free call packages apply long-term PR activity pattern.

- High PR activity: In high PR activity, the channel has long ON and short OFF periods. High PR activity level can be found in highly congested rush hours, where all the channels are mostly occupied.

- Low PR activity: In low PR activity, the channel has short ON and long OFF periods. Low PR activity level can be observed during less peak hours.

To achieve PUs activity for all channels, the rate parameter $\lambda_{x}$ and $\lambda_{y}$ of the exponential distributions are varied as indicated in Table 5 [36].

We performed extensive NS-2 simulations to present the performance analysis of the five channel selection strategies under varying PU node activity considering the most two vital performance metric: average interference ratio and spectrum opportunity utilization. The number of CR nodes is 100; the total channels for each CR are varied from 5 to 10 channels. The examined PU activity is applied to all the available channels similarly. Figure 17 shows the results of the average interference ratio for LITC, BFC, GA, ICSSSS, and ILFCS in case of low PU activity level. It is seen that, when number of channel is 5, ILFCS minimizes the interference ratio by $12 \%, 10 \%$, $4 \%$, and $2 \%$ compared to LITC, GA, BFC, and ICSSSS respectively. Besides, when number of available channel is 10 , ILFCS minimizes the interference ratio by $11 \%, 9 \%$, $4 \%$, and $2 \%$ compared to LITC, GA, BFC, and ICSSSS respectively.

Figure 18 shows the results of the average interference ratio for LITC, BFC, GA, ICSSSS, and ILFCS in case of long-term PU activity. It is seen that, when number of channel is 5, ILFCS decreases the interference ratio by $47 \%, 37 \%, 30 \%$, and $7 \%$ compared to LITC, GA, BFC, and

Table 5 PUs' activity patterns

\begin{tabular}{lllll}
\hline PU activity & ON & OFF & $\lambda_{x}$ & $\lambda_{y}$ \\
\hline Long-term activity & $\lambda_{x} \leq 1$ & $\lambda_{y} \leq 1$ & Long ON & Long OFF \\
High activity & $\lambda_{x} \leq 1$ & $\lambda_{y}>1$ & Long ON & Short OFF \\
Low activity & $\lambda_{x}>1$ & $\lambda_{y} \leq 1$ & Short ON & Long OFF \\
\hline
\end{tabular}

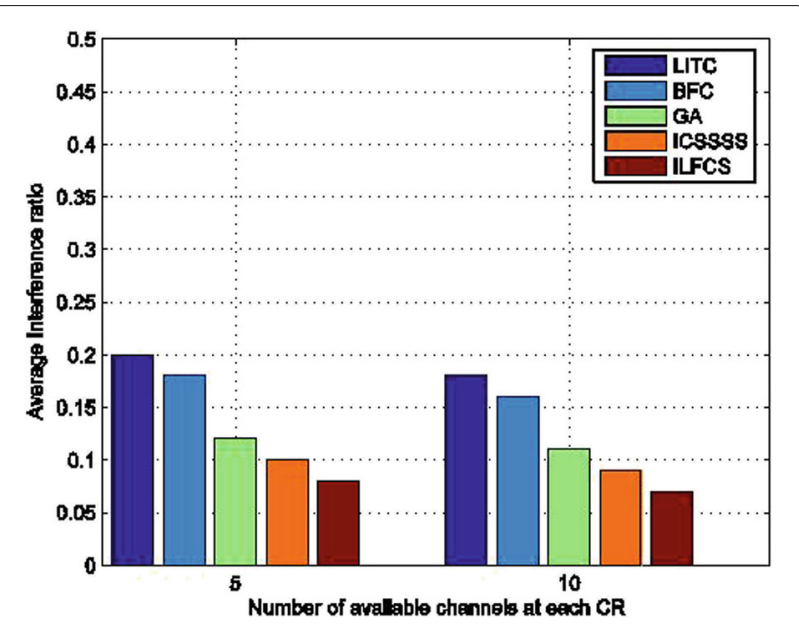

Fig. 17 Average interference ratio for LITC, BFC, GA, ICSSSS, and ILFCS in case of low PU activity. The results of different simulation experiments to measure average interference ratio for LITC, BFC, GA, ICSSSS, and ILFCS in case of low PU activity are shown

ICSSSS respectively. Moreover, when number of channel is 10 ILFCS decreases the interference ratio by $42 \%, 34 \%$, $28 \%$ and $5 \%$ compared to LITC, GA, BFC, and ICSSSS respectively.

Figure 19 shows the results of the average interference ratio for LITC, BFC, GA, ICSSSS, and ILFCS in case of high PU activity level. It is seen that, when the number of channels is 5, ILFCS decreases the interference ratio by $28 \%, 23 \%, 21 \%$ and $3 \%$ compared to LITC, GA, BFC, and ICSSSS respectively. Besides, when the number of channels is 5, ILFCS decreases the interference ratio by $28 \%, 23 \%, 20 \%$ and $3 \%$ compared to LITC, GA, BFC, and ICSSSS respectively.

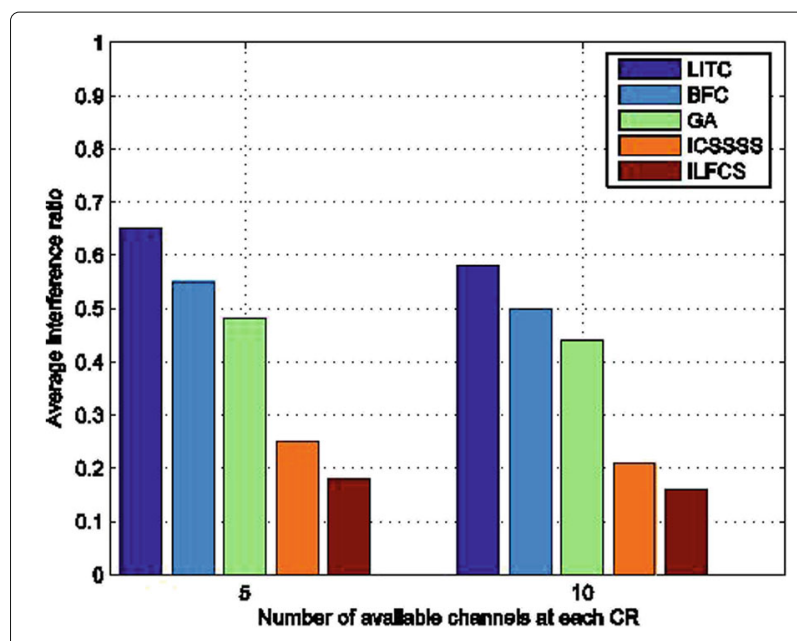

Fig. 18 Spectrum opportunity utilization for LITC, BFC, GA, ICSSSS, and ILFCS in case of long-term PU activity. Shows the results of different simulation experiments to measure Spectrum opportunity utilization for LITC, BFC, GA, ICSSSS, and ILFCS in case of long-term PU activity 


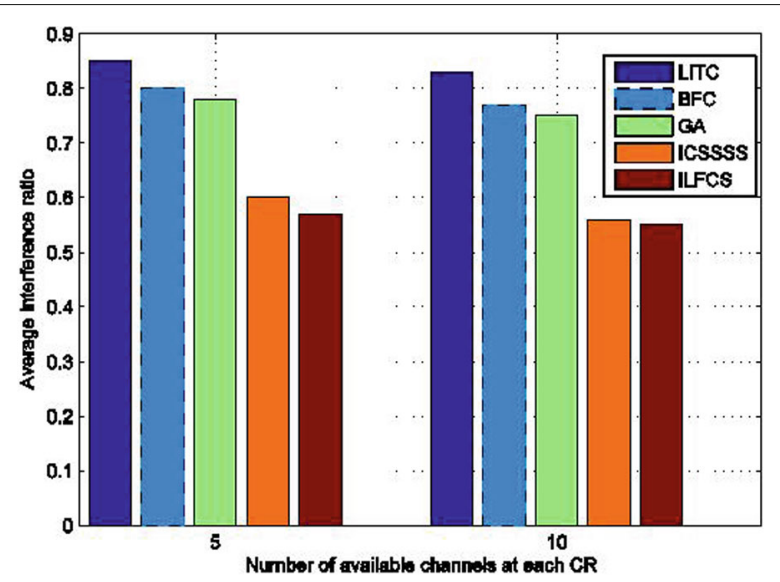

Fig. 19 Spectrum opportunity utilization for LITC, BFC, GA, ICSSSS, and ILFCS in case of high PU activity level. The results of different simulation experiments to measure spectrum opportunity utilization for LITC, BFC, GA, ICSSSS, and ILFCS in case of high PU activity level are shown

Figure 20 shows the results of spectrum opportunity utilization ratio for LITC, BFC, GA, ICSSSS, and ILFCS in case of low PU activity level. It is seen that, when number of channel is 5, ILFCS increases the spectrum opportunity utilization ratio by $12 \%, 10 \%, 4 \%$ and $2 \%$ compared to LITC, GA, BFC, and ICSSSS respectively. While as number of channel is 10, ILFCS increases the spectrum opportunity utilization ratio by $11 \%, 9 \%, 4 \%$, and $2 \%$ compared to LITC, GA, BFC, and ICSSSS respectively.

Figure 21 shows the results of the spectrum opportunity utilization ratio for LITC, BFC, GA, ICSSSS, and ILFCS in case of long-term PU activity. It is seen that ILFCS increases the spectrum opportunity utilization ratio by $45 \%, 35 \%, 28 \%$, and $5 \%$ compared to LITC, GA, BFC,

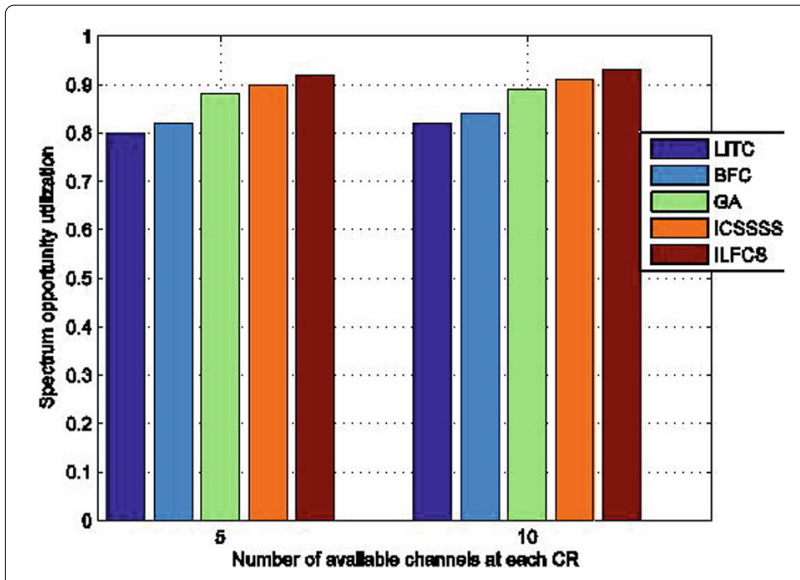

Fig. 20 Spectrum opportunity utilization for LITC, BFC, GA, ICSSSS, and ILFCS in case of low PU activity level. The results of different simulation experiments to measure Spectrum opportunity utilization for LITC, BFC, GA, ICSSSS, and ILFCS in case of low PU activity level are shown

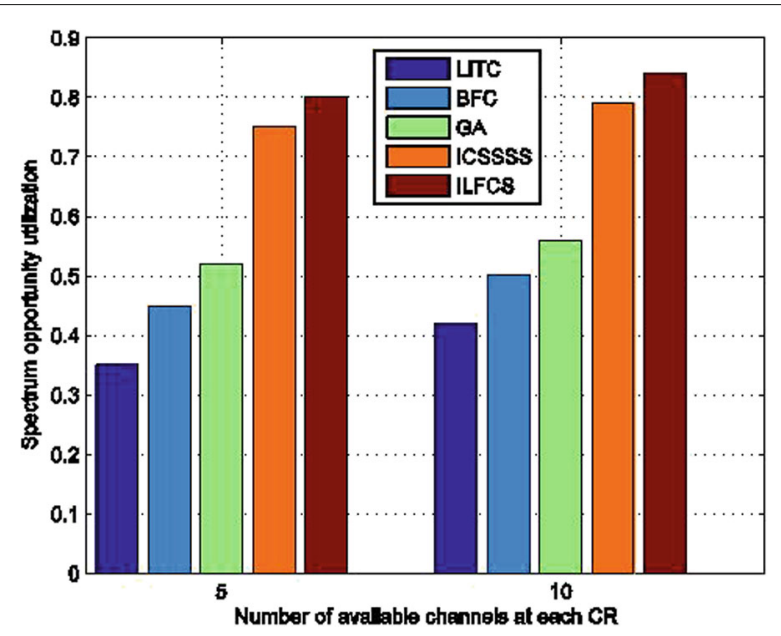

Fig. 21 Spectrum opportunity utilization for LITC, BFC, GA, ICSSSS, and ILFCS in case of long-term PU activity. The results of different simulation experiments to measure Spectrum opportunity utilization for LITC, BFC, GA, ICSSSS, and ILFCS in case of long-term PU activity are shown

and ICSSSS respectively. While as number of channel is 10, ILFCS increases the spectrum opportunity utilization ratio by $42 \%, 34 \%, 28 \%$, and $5 \%$ compared to LITC, GA, $\mathrm{BFC}$, and ICSSSS respectively.

Figure 22 shows the results of the spectrum opportunity utilization ratio for LITC, BFC, GA, ICSSSS, and ILFCS in case of high PU activity level. It is seen that ILFCS increases the spectrum opportunity utilization ratio by $28 \%, 25 \%, 23 \%$, and $3 \%$ compared to LITC, GA, BFC, and ICSSSS respectively. While as number of channel is 10, ILFCS increases the spectrum opportunity utilization ratio by $28 \%, 22 \%, 20 \%$, and $3 \%$ compared to LITC, GA, $\mathrm{BFC}$, and ICSSSS respectively.

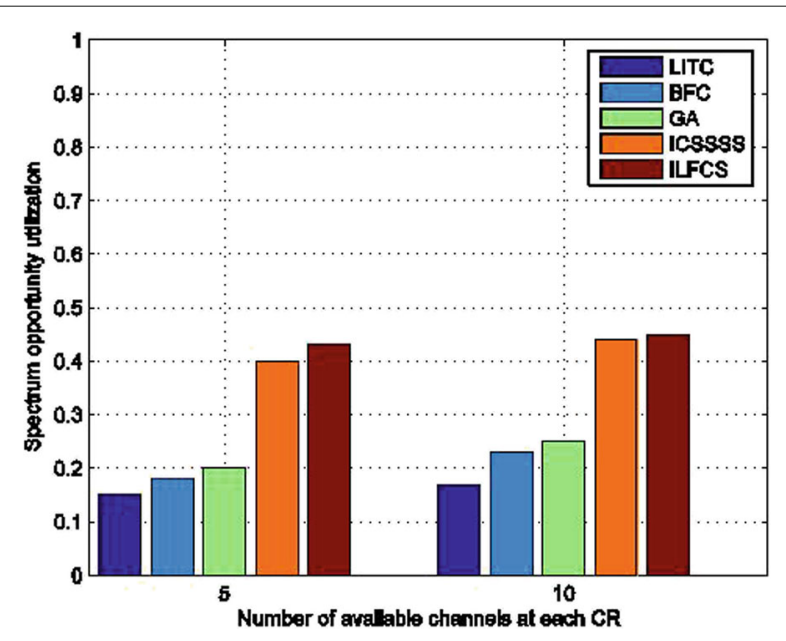

Fig. 22 Spectrum opportunity utilization for LITC, BFC, GA, ICSSSS, and ILFCS in case of high PU activity. The results of different simulation experiments to measure spectrum opportunity utilization for LITC, BFC, GA, ICSSSS, and ILFCS in case of high PU activity are shown 
As illustrated in Figs. 17, 18, 19, 20, 21, and 22, the proposed ILFCS outperforms LITC, BFC, GA, and ICSSSS strategies concerning different scenarios for PUs' activities. The minimum improvement for the ILFCS occurs in low-term PU activity pattern, because the duration time for OFF state is long. Therefore, the chance of interference is low, and consequently, the spectrum utilization is high. On the other hand, in case of long- and high-term PU activity, the maximum improvement occurs because the chance of interference is high due to the long duration of ON state.

\section{Conclusions}

In this paper, we consider the requirements for enhancing CRAHNs performance by designing an intelligent distributed channel selection strategy. An intelligent learning fuzzy-based channel selection framework for cognitive radio networks (ILFCS) is proposed. ILFCS aims to select the best channel which satisfies CRs' transmission requirements and resolves the problems of channel sharing. The key contribution of ILFCS is to avoid erroneous sensed channels by eliminating both false alarm and miss detection metrics. In ILFCS, sensing errors are handled by $K$-means algorithm to remove $\mathrm{ON}$ state channels and MD channels. This leads to select the best channel in an intelligent way. Consequently, CRs are allowed to transmit their packets without any interference with PUs or switching from channel to other which in turn reduces the average delay over the entire network. Furthermore, it can be concluded that ILFCS performance is enhanced with the increase of the number of existing channels. The low number of channels decreases the opportunity for CR users to find free channels for transmission. The performance of the proposed ILFCS is evaluated concerning the impact of changing CRs' density, number of channels and PU activity patterns. NS2 simulation results show that ILFCS significantly outperforms LITC, BFC, GA, and ICSSSS in terms of the average interference ratio, average throughput, end-to-end delay, packet delivery ratio, and spectrum opportunity utilization.

\footnotetext{
Abbreviations

ANFIS: Neuro-fuzzy inference adaptive system; BFC: Best fit channel selection; CCC: Common control channel; CR: Cognitive radio; CRAHN: Ad-Hoc network; CRCN: Cognitive radio cognitive network; CRNs: Cognitive radio networks; CRs: Radio users; EGSD: Energy-efficient game-theory-based spectrum decision; FA: False alarm ; FAHP: Fuzzy analytic hierarchy process; FCC: Federal communications commission; FIS: Fuzzy inference system; GC-DCA: Graph coloring-based dynamic channel allocation; GA: Genetic algorithm; IDSA: Intelligent fuzzy based dynamic spectrum allocation; ILFCS: Intelligent learning fuzzy based channel selection; ISSSS: Intelligent channel selection scheme using a self organized map followed by simple segregation; LITC: Longest idle time channel selection; MD: Miss detection; PUs: Primary users; QoS: Quality of service RF: Radio frequency; SINR: Signal-to-interference-plus-noise ratio
}

Funding

All funding resources are acknowledged.

\section{Authors' contributions}

The idea of this work was proposed by RA and AD. RA, AS, and MB performed the experiments and analyzed the simulation results. RA wrote the paper. All authors read and approved the final manuscript.

\section{Competing interests}

The authors declare that they have no competing interests.

\section{Publisher's Note}

Springer Nature remains neutral with regard to jurisdictional claims in published maps and institutional affiliations.

Received: 11 January 2018 Accepted: 1 October 2018

Published online: 20 October 2018

\section{References}

1. W. Huixin, M. Duo, L. He, Analysis and simulation of the dynamic spectrum allocation based on parallel immune optimization in cognitive wireless networks. Sci. World J. 2014, 8 (2014). Article ID 623670

2. H. Eltom, S. Kandeepan, R.J. Evans, Y.C. Liang, B. Ristic, Statistical spectrum occupancy prediction for dynamic spectrum access: a classification. EURASIP J. Wirel. Commun. Netw. 2018(1), 29 (2018)

3. W.-Y. Lee, I.F. Akyldiz, A spectrum decision framework for cognitive radio networks. IEEE Trans. Mob. Comput. 10(2), 161-174 (2011)

4. C. Wu, Y. Wang, Z. Yin, Energy-efficiency opportunistic spectrum allocation in cognitive wireless sensor network. EURASIP J. Wirel. Commun. Netw. 2018(1), 13 (2018)

5. W. Khalid, H. Yu, Optimal sensing performance for cooperative and non-cooperative cognitive radio networks. Int. J. Distrib. Sensor Netw. 13(11), 1550147717744992 (2017)

6. P. Liu, W. Qi, E. Yuan, L. Wei, Y. Zhao, Full-duplex cooperative sensing for spectrum-heterogeneous cognitive radio networks. Sensors. 17(8), 1773 (2017)

7. C. Fu, Y. Li, Y. He, M. Jin, G. Wang, P. Lei, An inter-frame dynamic double-threshold energy detection for spectrum sensing in cognitive radios. EURASIP J. Wirel. Commun. Netw. 2017(1), 118 (2017)

8. N. Giweli, S.A. Shahrestani, H.N. Cheung, Selection of spectrum sensing method to enhance qos in cognitive radio networks. Int. J. Wirel. Mob. Netw. 8(1), 39-50 (2016)

9. I.F. Akyildiz, B.F. Lo, R. Balakrishnan, Cooperative spectrum sensing in cognitive radio networks: A survey. Phys. Commun. 4(1), 40-62 (2011)

10. F. Hu, B. Chen, X. Zhai, C. Zhu, Channel selection policy in multi-su and multi-pu cognitive radio networks with energy harvesting for internet of everything. Mob. Inf. Syst. 2016, 12 (2016). Article ID 6024928

11. Y. Zhao, M. Song, C. Xin, A weighted cooperative spectrum sensing framework for infrastructure-based cognitive radio networks. Comput. Commun. 34(12), 1510-1517 (2011)

12. M.T. Masonta, M. Mzyece, N. Ntlatlapa, Spectrum decision in cognitive radio networks: a survey. IEEE Commun. Surv. Tutor. 15(3), 1088-1107 (2013)

13. L. Mpiana, K. Djouani, Y. Hamam, Optimized spectrum selection through instantaneous channels characteristics evaluation in cognitive radio. Procedia Comput. Sci. 94, 341-346 (2016)

14. J. Huang, X. Zeng, X. Tan, X. Jian, Y. He, Spectrum allocation for cognitive radio networks with non-deterministic bandwidth of spectrum hole. China Commun. 14(3), 87-96 (2017)

15. Y. Tang, D. Grace, T. Clarke, J. Wei, in 201111 th International Symposium on Communications \& Information Technologies (ISCIT). Multichannel non-persistent csma mac schemes with reinforcement learning for cognitive radio networks (IEEE, Hangzhou, 2011), pp. 502-506

16. H. Li, Multiagent-learning for aloha-like spectrum access in cognitive radio systems. EURASIP J. Wirel. Commun. Netw. 2010(1), 876216 (2010)

17. J. He, J. Peng, F. Jiang, G. Qin, W. Liu, A distributed q learning spectrum decision scheme for cognitive radio sensor network. Int. J. Distrib. Sensor Netw. 11(5), 301317 (2015)

18. J.R. Gállego, M. Canales, J. Ortín, Distributed resource allocation in cognitive radio networks with a game learning approach to improve aggregate system capacity. Ad Hoc Netw. 10(6), 1076-1089 (2012)

19. S. Salim, S. Moh, An energy-efficient game-theory-based spectrum decision scheme for cognitive radio sensor networks. Sensors. 16(7), 1009 (2016) 
20. B. Tushir, S.K. Dhurandher, I. Woungang, M.S. Obaidat, V. Teotia, in 2016 IEEE International Conference on Communications (ICC). Graph colouring technique for efficient channel allocation in cognitive radio networks (IEEE, Kuala Lumpur, 2016), pp. 1-5

21. L. Ding, T. Melodia, S.N. Batalama, J.D. Matyjas, M.J. Medley, Cross-layer routing and dynamic spectrum allocation in cognitive radio ad hoc networks. IEEE Trans. Veh. Technol. 59(4), 1969-1979 (2010)

22. H. Pareek, A. Singh, in International Conference on Recent Trends in Information, Telecommunication and Computing, ITC. An adaptive spectrum assignment algorithm in cognitive radio network (ACEEE, 2014), pp. $408-418$

23. W. Yang, X. Zhao, Robust resource allocation for cognitive relay networks with multiple primary users. EURASIP J. Wirel. Commun. Netw. 2017(1), 107 (2017)

24. T. Veeramakali, S. Jayashri, S. Prabu, Intelligent dynamic spectrum allocation with bandwidth flexibility in cognitive radio network. Clust. Comput. 20(2), 1575-1586 (2017)

25. D.A. López, C.A. Rojas, D.F. Zapata, E.R. Trujillo, Designing a mac algorithm for equitable spectrum allocation in cognitive radio wireless networks. Wirel. Pers. Commun. 98(1), 363-394 (2018)

26. J. Elhachmi, Z. Guennoun, Cognitive radio spectrum allocation using genetic algorithm. EURASIP J. Wirel. Commun. Netw. 2016(1), 133 (2016)

27. S. Bayhan, F. Alagöz, Distributed channel selection in crahns: A non-selfish scheme for mitigating spectrum fragmentation. Ad Hoc Netw. 10(5), 774-788 (2012)

28. L. Yang, L. Cao, H. Zheng, Proactive channel access in dynamic spectrum networks. Phys. Commun. 1(2), 103-111 (2008)

29. M. Hoyhtya, S. Pollin, A. Mammela, in 2008 First International Workshop on Cognitive Radio and Advanced Spectrum Management. Performance improvement with predictive channel selection for cognitive radios (IEEE, Aalborg, 2008), pp. 1-5

30. T.M. Salem, S. Abdel-Mageid, S.M. Abdel-Kader, M. Zaki, Icssss: An intelligent channel selection scheme for cognitive radio ad hoc networks using a self organized map followed by simple segregation. Pervasive Mob. Comput. 39, 195-213 (2017)

31. R. Hussain, A. Shakeel, A. lqbal, J. Ahmed, S. Alvi, Q. ul. Hasan, S.A. Malik, et al., Efficient idle channel discovery mechanism through cooperative parallel sensing in cognitive radio network. EURASIP J. Wirel. Commun. Netw. 2018(1), 75 (2018)

32. M. Çamurli, D. Gözüpek, in Ad Hoc Networks. ADHOCNETS 2015. Lecture Notes of the Institute for Computer Sciences, Social Informatics and Telecommunications Engineering, book series (LNICST, volume 140), ed. by N. Mitton, A. Gallais, M. Kantarci, and S. Papavassiliou. Channel switching cost-aware resource allocation for multi-hop cognitive radio networks with a single transceiver (Springer, Cham, 2014), pp. 158-168

33. S. Ping, A. Aijaz, O. Holland, A.-H. Aghvami, Sacrp: A spectrum aggregation-based cooperative routing protocol for cognitive radio ad-hoc networks. IEEE Trans. Commun. 63(6), 2015-2030 (2015)

34. C.-M. Chao, Y.-T. Ling, C.-H. Jiang, Number of channels adjustment for cognitive radio networks. J. Netw. Comput. Appl. 96, 1-13 (2017)

35. M. Zareei, A. Islam, S. Baharun, C. Vargas-Rosales, L. Azpilicueta, N. Mansoor, Medium access control protocols for cognitive radio ad hoc networks: A survey. Sensors. 17(9), 2136 (2017)

36. M.H. Rehmani, A.C. Viana, H. Khalife, S. Fdida, Surf: A distributed channel selection strategy for data dissemination in multi-hop cognitive radio networks. Comput. Commun. 36(10-11), 1172-1185 (2013)

37. N.O.F. Elssied, O. Ibrahim, W. Abu-Ulbeh, An improved of spam e-mail classification mechanism using k-means clustering. J. Theor. Appl. Inf. Technol. 60(3), 568-580 (2014)

38. P. Chanak, I. Banerjee, Fuzzy rule-based faulty node classification and management scheme for large scale wireless sensor networks. Expert Syst. Appl. 45, 307-321 (2016)

39. S. Tabatabaei, F. Hosseini, A fuzzy logic-based fault tolerance new routing protocol in mobile ad hoc networks. Int. J. Fuzzy. Syst. 18(5), 883-893 (2016)

40. E.H. Mamdani, S. Assilian, An experiment in linguistic synthesis with a fuzzy logic controller. Int. J. Man-machine Stud. 7(1), 1-13 (1975)

41. P. Nayak, A. Devulapalli, A fuzzy logic-based clustering algorithm for wsn to extend the network lifetime. IEEE Sensors. J. 16(1), 137-144 (2016)

42. F. Hu, S. Kumar, Multimedia over Cognitive Radio Networks: Algorithms, Protocols, and Experiments. (CRC Press, London, 2014)

\section{Submit your manuscript to a SpringerOpen ${ }^{\circ}$ journal and benefit from:}

- Convenient online submission

Rigorous peer review

- Open access: articles freely available online

- High visibility within the field

- Retaining the copyright to your article

Submit your next manuscript at $>$ springeropen.com 